\title{
THE AUSLANDER-REITEN TRANSLATION IN SUBMODULE CATEGORIES
}

\author{
CLAUS MICHAEL RINGEL AND MARKUS SCHMIDMEIER
}

Dedicated to Idun Reiten on the occasion of her $60^{\text {th }}$ birthday

\begin{abstract}
Let $\Lambda$ be an artin algebra or, more generally, a locally bounded associative algebra, and $\mathcal{S}(\Lambda)$ the category of all embeddings $(A \subseteq B)$ where $B$ is a finitely generated $\Lambda$-module and $A$ is a submodule of $B$. Then $\mathcal{S}(\Lambda)$ is an exact Krull-Schmidt category which has Auslander-Reiten sequences. In this manuscript we show that the Auslander-Reiten translation in $\mathcal{S}(\Lambda)$ can be computed within mod $\Lambda$ by using our construction of minimal monomorphisms. If in addition $\Lambda$ is uniserial, then any indecomposable nonprojective object in $\mathcal{S}(\Lambda)$ is invariant under the sixth power of the Auslander-Reiten translation.
\end{abstract}

Let $\Lambda$ be an artin algebra, and $\bmod \Lambda$ the category of finitely generated $\Lambda$ modules (these are just the $\Lambda$-modules of finite length). The homomorphism category $\mathcal{H}(\Lambda)$ has as objects the maps $f$ in $\bmod \Lambda$, and morphisms are given by commutative diagrams. In this paper, we draw attention to the full subcategory $\mathcal{S}(\Lambda)$ of $\mathcal{H}(\Lambda)$ of all monomorphisms (or subobjects), but also to the corresponding subcategory $\mathcal{F}(\Lambda)$ of $\mathcal{H}(\Lambda)$ of all epimorphisms (or factor objects). Categories of the form $\mathcal{S}(\Lambda)$ are much more complicated than the underlying module categories $\bmod \Lambda$; for example, if $\Lambda$ is a uniserial ring, then $\Lambda$ is of finite representation type, whereas the category $\mathcal{S}(\Lambda)$ may have finitely or infinitely many indecomposable objects, or even be of wild representation type, depending on the Loewy length of $\Lambda$. Since Garrett Birkhoff in 1934 proposed the study of such submodule categories, they have proven to provide a rich source for classification problems, and to attract the use of methods from various areas of algebra including representations of finite dimensional algebras, lattices over tiled orders, representations of posets, and matrix classification. In this manuscript we intend to lay the foundation for an Auslander-Reiten type theory of submodule categories. Here is a preview:

If we consider a map $f: A \rightarrow B$ of $\Lambda$-modules as an object of $\mathcal{H}(\Lambda)$, we will write either

$$
(A \stackrel{f}{\rightarrow} B) \quad \text { or } \quad \stackrel{A}{\downarrow f}, \quad
$$

but often also just $f$, whatever will be convenient and not misleading. The category $\mathcal{H}(\Lambda)$ is an abelian category, in fact it is equivalent to the category of finitely generated modules over the triangular matrix ring $\left(\begin{array}{ll}\Lambda & \Lambda \\ 0 & \Lambda\end{array}\right)$, and hence $\mathcal{S}(\Lambda)$ as well as $\mathcal{F}(\Lambda)$ are exact Krull-Schmidt categories. We determine the projective and the

Received by the editors April 30, 2005 and, in revised form, September 30, 2005.

2000 Mathematics Subject Classification. Primary 16G70; Secondary 18E30.

Key words and phrases. Auslander-Reiten sequences, approximations, triangulated categories. 
injective objects in $\mathcal{S}(\Lambda)$ and $\mathcal{F}(\Lambda)$. For example, if $I$ is an indecomposable injective $\Lambda$-module, then

$$
(0 \rightarrow I) \quad \text { and } \quad(I \stackrel{1}{\rightarrow} I)
$$

are both indecomposable injective objects in $\mathcal{S}(\Lambda)$, but clearly the first is not injective in $\mathcal{H}(\Lambda)$ (Proposition 1.4).

In order to see that $\mathcal{S}(\Lambda)$ has Auslander-Reiten sequences, we only have to show that $\mathcal{S}(\Lambda)$ is functorially finite in $\mathcal{H}(\Lambda)$, according to Auslander and Smalø (Theorem 2.4 in $[\mathrm{AS}]$ ). But this is easy: In the abelian category $\bmod \Lambda$, every map $f: A \rightarrow B$ can be factorized as the composition of an epimorphism $\operatorname{Epi}(f)$ and a monomorphism Mono $(f)$. The factorization yields a morphism $f \rightarrow \operatorname{Mono}(f)$ in $\mathcal{H}(\Lambda)$, and this morphism is a minimal left $\mathcal{S}(\Lambda)$-approximation for the object $f \in \mathcal{H}(\Lambda)$. To obtain a minimal right $\mathcal{S}(\Lambda)$-approximation for $f$, let $e^{\prime}: \operatorname{Ker}(f) \rightarrow$ $\mathrm{I} \operatorname{Ker}(f)$ be an injective envelope and choose an extension $e: A \rightarrow \operatorname{IKer}(f)$ of $e^{\prime}$. We call the map

$$
\operatorname{Mimo}(f)=\left[\begin{array}{ll}
f & e
\end{array}\right]: \quad A \rightarrow B \oplus \operatorname{IKer}(f)
$$

a minimal monomorphism for $f$. In this way, we obtain a morphism $\operatorname{Mimo}(f) \rightarrow f$ in the category $\mathcal{H}(\Lambda)$, and this morphism turns out to be the desired minimal right $\mathcal{S}(\Lambda)$-approximation for $f \in \mathcal{H}(\Lambda)$ (Proposition 2.4). Thus, the category $\mathcal{S}(\Lambda)$ is functorially finite in $\mathcal{H}(\Lambda)$ and hence has Auslander-Reiten sequences. Note that $\mathcal{S}(\Lambda)$-approximations provide recipes for calculating relative Auslander-Reiten sequences in the subcategory $\mathcal{S}(\Lambda)$ as soon as Auslander-Reiten sequences are known in $\mathcal{H}(\Lambda)$.

In the module category $\mathcal{H}(\Lambda)$, Auslander-Reiten translates are computed via the Nakayama functor, as usual. Using approximations and the equivalence between $\mathcal{S}(\Lambda)$ and $\mathcal{F}(\Lambda)$ given by the kernel and cokernel functors, corresponding AuslanderReiten sequences are obtained for the categories $\mathcal{S}(\Lambda)$ and $\mathcal{F}(\Lambda)$; see Proposition 3.2. (Surprisingly the converse is also true: Auslander-Reiten sequences in $\mathcal{S}(\Lambda)$ and $\mathcal{F}(\Lambda)$ give rise to such sequences in the category $\mathcal{H}(\Lambda)$; see Proposition 3.5.)

Revisiting the construction of minimal monomorphisms, we show that if two morphisms $f, g: A \rightarrow B$ in $\bmod \Lambda$ differ only by a map which factorizes through an injective $\Lambda$-module, and if $B$ has no nonzero injective direct summands, then $\operatorname{Mimo}(f)$ and $\operatorname{Mimo}(g)$ are isomorphic objects in $\mathcal{S}(\Lambda)$ (Proposition 4.1).

Our key result is this: For an indecomposable nonprojective object $(A \stackrel{f}{\rightarrow} B)$ in $\mathcal{S}(\Lambda)$, the Auslander-Reiten translate $\tau_{\mathcal{S}}(f)$ can be computed directly within the category $\bmod \Lambda$. This is done as follows: Let $g: B \rightarrow C$ be the cokernel of $f$. Recall that the Auslander-Reiten translation in $\bmod \Lambda$ gives rise to a functor $\tau_{\Lambda}: \underline{\bmod } \Lambda \rightarrow \overline{\bmod } \Lambda$ where $\underline{\bmod } \Lambda$ and $\overline{\bmod } \Lambda$ denote the factor categories of $\bmod \Lambda$ modulo all maps which factorize through a projective or through an injective $\Lambda$ module, respectively. Next take a representative $h: D \rightarrow E$ for the morphism $\tau_{\Lambda}(g)$ such that $D$ and $E$ have no nonzero injective direct summands. As $h$ is determined uniquely, up to a map which factorizes through an injective module, we will see that $\operatorname{Mimo}(h)$ is determined uniquely, up to isomorphism, as an object in $\mathcal{S}(\Lambda)$. This is the Auslander-Reiten translate $\tau_{\mathcal{S}}(f)$ for $f$ in the category $\mathcal{S}(\Lambda)$ (Theorem 5.1). Thus we may write:

$$
\tau_{\mathcal{S}}(f)=\operatorname{Mimo} \tau_{\Lambda} \operatorname{Cok}(f) .
$$


If $\Lambda$ is a self-injective algebra, the stable category $\underline{\bmod } \Lambda=\overline{\bmod } \Lambda$ has the structure of a triangulated category. We observe that if $A \stackrel{\bar{f}}{\rightarrow} B \stackrel{\bar{g}}{\rightarrow} C \stackrel{\bar{h}}{\rightarrow} \Omega^{-1} A$ is a triangle (with $\Omega^{-1}$ the suspension functor) and $f$ is a map representing $\bar{f}$, then a map $g$ representing the rotate $\bar{g}$ is obtained as $g=\operatorname{Cok} \operatorname{Mimo}(f)$. The functor $\tau_{\Lambda}$ commutes with $\Omega^{-1}$ and with the rotation $\bar{f} \mapsto \bar{g}$ in a triangle, and as a consequence, the formulae

$$
\overline{\tau_{\mathcal{S}}^{3}(f)} \cong-\tau_{\Lambda}^{3} \Omega^{-1}(\bar{f}) \text { and } \overline{\tau_{\mathcal{S}}^{6}(f)} \cong \tau_{\Lambda}^{6} \Omega^{-2}(\bar{f})
$$

hold (Theorem 6.2). In particular, if $\tau_{\Lambda}$ coincides with $\Omega^{2}$, it follows that

$$
\tau_{\mathcal{S}}^{3}(f) \cong-\operatorname{Mimo} \Omega^{5}(f)
$$

for any indecomposable nonprojective object $f$ in $\mathcal{S}(\Lambda)$ (Corollary 6.4). For example, in the special case that $\Lambda$ is a commutative uniserial ring, all the functors $\tau_{\Lambda}$, $\Omega^{2}$ and $\Omega^{-2}$ are equivalent to the identity functor on $\underline{\bmod } \Lambda$ and then the formula yields

$$
\tau_{\mathcal{S}}^{6}(f) \cong f
$$

for $f$ an indecomposable nonprojective object in $\mathcal{S}(\Lambda)$ (Corollary 6.5).

Let $\left(C^{\prime} \stackrel{c}{\rightarrow} C\right)$ be an indecomposable nonprojective object in $\mathcal{S}(\Lambda)$. The Auslander-Reiten sequence

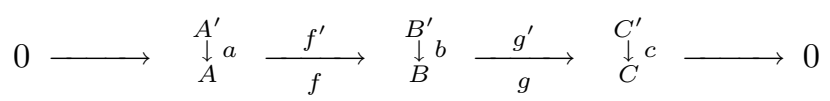

in $\mathcal{S}(\Lambda)$ is made up from two short exact sequences in $\bmod \Lambda$ given by the sequence $0 \rightarrow A^{\prime} \stackrel{f^{\prime}}{\rightarrow} B^{\prime} \stackrel{g^{\prime}}{\rightarrow} C^{\prime} \rightarrow 0$ of the submodules and the sequence $0 \rightarrow$ $A \stackrel{f}{\rightarrow} B \stackrel{g}{\rightarrow} C \rightarrow 0$ of the big modules. By Proposition 7.2, these two sequences are "usually" split exact. We list the exceptions and collect our findings about the structure of the middle term $\left(b: B^{\prime} \rightarrow B\right)$.

Let us stress that the categories $\bmod \Lambda, \mathcal{H}(\Lambda)$ and $\mathcal{S}(\Lambda)$ usually behave very differently. Consider for example the case of $\Lambda=\Lambda_{n}=k[T] / T^{n}$. These $k$-algebras $\Lambda_{n}$ are all of finite representation type: indeed, there is (up to isomorphism) precisely one indecomposable $\Lambda$-module of length $i$, for $1 \leq i \leq n$, and these are all the indecomposables. On the other hand, it is well-known that the matrix ring $\left(\begin{array}{cc}\Lambda_{n} & \Lambda_{n} \\ 0 & \Lambda_{n}\end{array}\right)$ is representation finite only for $n \leq 3$, thus, for $n \geq 4$ there are infinitely many isomorphism classes of indecomposable objects in $\mathcal{H}\left(\Lambda_{n}\right)$; see for example [S, Section 2]. For $n \leq 5$, the subcategory $\mathcal{S}\left(\Lambda_{n}\right)$ of $\mathcal{H}\left(\Lambda_{n}\right)$ consists of only finitely many isomorphism classes of indecomposable objects, whereas there are infinitely many isomorphism classes of indecomposable objects in $\mathcal{S}\left(\Lambda_{n}\right)$, for any $n \geq 6$; see [RS1]. One may consult these references and also [RS2] for proofs that the categories $\mathcal{H}\left(\Lambda_{4}\right)$ and $\mathcal{S}\left(\Lambda_{6}\right)$ are "tame", whereas the categories $\mathcal{H}\left(\Lambda_{n}\right)$ for $n \geq 5$ and $\mathcal{S}\left(\Lambda_{n}\right)$ for $n \geq 7$ are "wild".

Notation. The condition that $\Lambda$ is an artin algebra can be weakened to require that $\Lambda$ be a locally bounded associative $k$-algebra or a locally bounded $k$-spectroid [GR]; then also coverings of finite dimensional algebras are included. We recall the corresponding definitions:

Let $k$ be a commutative local artinian ring and $\Lambda$ an associative $k$-algebra which need not have a unit element, but it is required that $\Lambda$ equals the $k$-space $\Lambda^{2}$ of all possible linear combinations of products in $\Lambda$. By $\bmod \Lambda$ we denote the category of all $\Lambda$-modules $B$ which have finite length when considered as $k$-modules and which 
are unitary in the sense that $\Lambda B=B$ holds. The algebra $\Lambda$ is said to be locally bounded if there is a complete set $\left\{e_{i}: i \in I\right\}$ of pairwise orthogonal primitive idempotents such that each of the indecomposable projective modules $e_{i} \Lambda$ and $\Lambda e_{i}$ has finite length as a $k$-module, for $i \in I$. If $\Lambda$ is locally bounded such that each indecomposable projective $\Lambda$-module is injective and each indecomposable injective $\Lambda$-module is projective, then we say that $\Lambda$ is a self-injective algebra. An artin algebra $\Lambda$ is called uniserial if both modules $\Lambda_{\Lambda}$ and ${ }_{\Lambda} \Lambda$ have unique composition series. In this case, all one-sided ideals are two-sided, namely the powers of the unique maximal ideal $\mathbf{m}=\operatorname{Rad} \Lambda$.

For the terminology around almost split morphisms we refer the reader to [ARS] and $[\mathrm{AS}]$. Here we use the term "Auslander-Reiten sequence" for "almost split sequence" and abbreviate "left (right) minimal almost split map" to "source (sink) map". The Auslander-Reiten translation in a category $\mathcal{C}$ is denoted by $\tau_{\mathcal{C}}$, but in case $\mathcal{C}=\bmod \Lambda$, we write $\tau_{\Lambda}$.

Finally, we want to apologize that our use of brackets when applying functions and functors is not at all consistent. We have inserted brackets whenever we felt that this improves the readability, but we have avoided multiple brackets whenever possible.

This paper was written in 2001 as a general introduction to a proposed volume devoted to the Birkhoff problem (dealing with subgroups of finite abelian groups as well as with invariant subspaces of linear operators). Unfortunately, we had to delay the Birkhoff project. Since the paper seems of interest in its own right, we now have decided to publish it independently. The authors are indebted to the referee, and also to Aslak Bakke Buan and Øyvind Solberg, for helpful remarks concerning the presentation of the results.

\section{Projective And injective objects}

In this section we determine the projective and the injective objects in the categories $\mathcal{S}(\Lambda)$ and $\mathcal{F}(\Lambda)$ and their associated sink and source maps. The injective objects in $\mathcal{S}(\Lambda)$ are also called "relatively injective" or "Ext-injective", as they may not be injective in the category $\mathcal{H}(\Lambda)$. Dually, the projective objects in $\mathcal{F}(\Lambda)$ may not be projective when considered as objects in $\mathcal{H}(\Lambda)$.

Let $\Lambda$ be an associative locally bounded $k$-algebra, and let $U(\Lambda)=\left(\begin{array}{ll}\Lambda & \Lambda \\ 0 & \Lambda\end{array}\right)$ be the associative $k$-algebra of upper triangular matrices with coefficients in $\Lambda$. First we recall well-known facts about $U(\Lambda)$ and about the category $\mathcal{H}(\Lambda)$ of morphisms between $\Lambda$-modules of finite $k$-length.

Lemma 1.1 (Basic facts about $\mathcal{H}(\Lambda)$ ).

1. The k-algebra $U(\Lambda)$ is locally bounded.

2. The $k$-categories $\bmod U(\Lambda)$ and $\mathcal{H}(\Lambda)$ are equivalent.

3. $\mathcal{H}(\Lambda)$ is an abelian Krull-Schmidt category.

4. Each object in $\mathcal{H}(\Lambda)$ has a projective cover and an injective envelope.

5. The category $\mathcal{H}(\Lambda)$ has Auslander-Reiten sequences.

The categories $\mathcal{S}(\Lambda)$ and $\mathcal{F}(\Lambda)$ are defined to be the full subcategories of $\mathcal{H}(\Lambda)$ which consist of all objects $(A \stackrel{f}{\rightarrow} B)$ in $\mathcal{H}(\Lambda)$ for which $f$ is a monomorphism or an epimorphism, respectively. These three categories are related by the kernel and 
cokernel functors:

$$
\begin{array}{llll}
\text { Cok }: & \mathcal{H}(\Lambda) \rightarrow \mathcal{F}(\Lambda), & (A \stackrel{f}{\rightarrow} B) \mapsto(B \stackrel{\text { can }}{\longrightarrow} \operatorname{Cok}(f)), \\
\text { Ker : } & \mathcal{H}(\Lambda) \rightarrow \mathcal{S}(\Lambda), \quad & (B \stackrel{g}{\rightarrow} C) \mapsto(\operatorname{Ker}(g) \stackrel{\text { incl }}{\longrightarrow} B) .
\end{array}
$$

Lemma 1.2 (Basic properties of $\mathcal{S}(\Lambda)$ and $\mathcal{F}(\Lambda)$ ).

1. With the exact structure given by the category $\mathcal{H}(\Lambda)$, the categories $\mathcal{S}(\Lambda)$ and $\mathcal{F}(\Lambda)$ are exact Krull-Schmidt $k$-categories.

2. The category $\mathcal{S}(\Lambda)$ is closed under kernels while $\mathcal{F}(\Lambda)$ is closed under cokernels. Both categories are closed under extensions.

3. The restrictions of the kernel and cokernel functors

$$
\text { Ker }: \mathcal{F}(\Lambda) \rightarrow \mathcal{S}(\Lambda) \quad \text { and } \quad \text { Cok }: \mathcal{S}(\Lambda) \rightarrow \mathcal{F}(\Lambda)
$$

induce a pair of inverse equivalences.

The equivalence between $\mathcal{S}(\Lambda)$ and $\mathcal{F}(\Lambda)$ is useful to deduce the structure of the projective and the injective objects in either of the two categories from the structure of the corresponding modules in $\mathcal{H}(\Lambda)$ which are described in the following

Lemma 1.3 (Projective and injective modules in $\mathcal{H}(\Lambda)$; the Nakayama functor).

$\mathrm{P}-1$. Let $P$ be an indecomposable projective $\Lambda$-module with radical $\operatorname{Rad} P$. The objects $(0 \rightarrow P)$ and $\left(1_{P}: P \rightarrow P\right)$ are indecomposable projective objects and have as sink maps the inclusions

$$
(0 \rightarrow \operatorname{Rad} P) \rightarrow(0 \rightarrow P) \quad \text { and } \quad(\operatorname{Rad} P \stackrel{\text { incl }}{\longrightarrow} P) \rightarrow(P \stackrel{1}{\rightarrow} P),
$$

respectively.

P-2. Each indecomposable projective object arises in this way.

I-1. Let I be an indecomposable injective $\Lambda$-module with socle Soc $I$. The indecomposable injective objects $(I \rightarrow 0)$ and $\left(1_{I}: I \rightarrow I\right)$ have as source maps the canonical maps

$$
(I \rightarrow 0) \rightarrow(I / \operatorname{Soc} I \rightarrow 0) \quad \text { and } \quad(I \stackrel{1}{\rightarrow} I) \rightarrow(I \stackrel{\text { can }}{\longrightarrow} I / \operatorname{Soc} I),
$$

respectively.

I-2. Each indecomposable injective object arises in this way.

$\mathrm{N}-1$. The operation of the Nakayama functor $\nu_{\mathcal{H}}$ on $\mathcal{H}(\Lambda)$ can be expressed in terms of the Nakayama functor $\nu=\nu_{\Lambda}$ in $\bmod \Lambda$. For $P$ a projective $\Lambda$-module we have

$$
\nu_{\mathcal{H}}(0 \rightarrow P)=(\nu P \stackrel{1}{\rightarrow} \nu P), \quad \nu_{\mathcal{H}}(P \stackrel{1}{\rightarrow} P)=(\nu P \rightarrow 0) .
$$

$\mathrm{N}-2$. On morphisms, the Nakayama functor $\nu_{\mathcal{H}}$ is given in the obvious way; for example if $(0, f):(0 \rightarrow P) \rightarrow\left(1_{Q}: Q \rightarrow Q\right)$ is a morphism between projective objects, then $\nu_{\mathcal{H}}(0, f)=(\nu f, 0):\left(1_{\nu P}: \nu P \rightarrow \nu P\right) \rightarrow(\nu Q \rightarrow 0)$.

We can now describe the projective and the injective objects in $\mathcal{S}(\Lambda)$.

Proposition 1.4 (Projective and injective objects in $\mathcal{S}(\Lambda)$ ).

P. The projective objects in $\mathcal{S}(\Lambda)$ and their sink maps are as in Lemma 1.3-P.

I-1. Let $I$ be an indecomposable injective $\Lambda$-module. Then the map $\left(1_{I}: I \rightarrow I\right)$ is an indecomposable injective object in $\mathcal{S}(\Lambda)$ and has as a source map the canonical $\operatorname{map}\left(1_{I}: I \rightarrow I\right) \rightarrow\left(1_{I / \operatorname{Soc} I}: I / \operatorname{Soc} I \rightarrow I / \operatorname{Soc} I\right)$.

I-2. If $I$ is an indecomposable injective $\Lambda$-module, then $(0 \rightarrow I)$ is indecomposable (relatively) injective in $\mathcal{S}(\Lambda)$ and has as a source map the inclusion $(0 \rightarrow I) \rightarrow$ (incl : Soc $I \rightarrow I$ ).

I-3. Each indecomposable (relatively) injective object in $\mathcal{S}(\Lambda)$ arises in this way. 
Proof. The projective modules in $\mathcal{H}(\Lambda)$ and their sink maps are objects and morphisms in the category $\mathcal{S}(\Lambda)$, hence the statement in Lemma 1.3-P holds for $\mathcal{S}(\Lambda)$. Similarly, the injective modules in $\mathcal{H}(\Lambda)$ and their source maps are in the category $\mathcal{F}(\Lambda)$, so the statement in 1.3-I holds for $\mathcal{F}(\Lambda)$. By applying the kernel functor from item 3 in Lemma 1.2 we obtain the objects and morphisms in I-1 and I-2. Since $\mathcal{H}(\Lambda)$ has sufficiently many projective and injective objects, so does $\mathcal{S}(\Lambda)$.

Let us add the dual statement for the category $\mathcal{F}(\Lambda)$. Of course, we know from Lemma 1.2 that the categories $\mathcal{S}(\Lambda)$ and $\mathcal{F}(\Lambda)$ are equivalent, thus there is no intrinsic need to deal with both categories separately. On the other hand, it may be useful for further references to have the precise formulations available.

Proposition 1.5 (Projective and injective objects in $\mathcal{F}(\Lambda)$ ).

$\mathrm{P}-1$. Let $P$ be an indecomposable projective $\Lambda$-module. Then the map $\left(1_{I}\right.$ : $P \rightarrow P)$ is an indecomposable projective object in $\mathcal{F}(\Lambda)$ and has as a sink map the inclusion $\left(1_{\operatorname{Rad} P}: \operatorname{Rad} P \rightarrow \operatorname{Rad} P\right) \rightarrow\left(1_{P}: P \rightarrow P\right)$.

$\mathrm{P}-2$. If $P$ is an indecomposable projective $\Lambda$-module, then $(P \rightarrow 0)$ is indecomposable (relatively) projective in $\mathcal{F}(\Lambda)$ with a sink map the canonical map $($ can $: P \rightarrow P / \operatorname{Rad} P) \rightarrow(P \rightarrow 0)$.

$\mathrm{P}-3 . \quad$ Each indecomposable (relatively) projective object in $\mathcal{S}(\Lambda)$ arises in this way.

I. The injective objects in $\mathcal{F}(\Lambda)$ and their source maps are as in Lemma 1.3-I.

Example. Consider the case that $\Lambda$ is uniserial with maximal ideal $\mathbf{m}$ and Loewy length $n \geq 2$. In $\mathcal{S}(\Lambda)$, there are two projective indecomposable objects, namely $P_{1}=(\Lambda=\Lambda)$ and $P_{2}=(0 \subseteq \Lambda)$; their sink maps are the inclusions

$$
(\mathbf{m} \subseteq \Lambda) \quad \rightarrow \quad P_{1} \quad \text { and } \quad(0 \subseteq \mathbf{m}) \quad \rightarrow \quad P_{2},
$$

respectively. One of them, the module $P_{1}=I_{1}$ is also injective, both in $\mathcal{H}(\Lambda)$ and in $\mathcal{S}(\Lambda)$, and has a source map the canonical map

$$
I_{1} \quad \rightarrow \quad\left(\Lambda / \mathbf{m}^{n-1}=\Lambda / \mathbf{m}^{n-1}\right) ;
$$

the second projective $P_{2}=I_{2}$ is relatively injective in $\mathcal{S}(\Lambda)$, and its source map is given by the inclusion

$$
I_{2} \quad \rightarrow \quad\left(\mathbf{m}^{n-1} \subseteq \Lambda\right)
$$

\section{LEFT AND RIGHT APPROXIMATIONS}

The categories $\mathcal{S}(\Lambda)$ and $\mathcal{F}(\Lambda)$ are functorially finite in $\mathcal{H}(\Lambda)$ and hence have Auslander-Reiten sequences. To show this, we determine the left and the right approximation for each object in $\mathcal{H}(\Lambda)$, in each of the subcategories $\mathcal{S}(\Lambda)$ and $\mathcal{F}(\Lambda)$.

Definitions. Let $\mathcal{S}$ be a subcategory of a module category $\mathcal{C}$, and $C \in \mathcal{C}$. A morphism $f: C \rightarrow S$ with $S \in \operatorname{add} \mathcal{S}$ is a left approximation of $C$ in $\mathcal{S}$ if the map

$$
\operatorname{Hom}\left(f, 1_{S^{\prime}}\right): \operatorname{Hom}_{\mathcal{C}}\left(S, S^{\prime}\right) \rightarrow \operatorname{Hom}_{\mathcal{C}}\left(C, S^{\prime}\right)
$$

is onto for each $S^{\prime} \in \mathcal{S}$. Moreover, $f$ is left minimal if each endomorphism $s \in$ $\operatorname{End}_{\mathcal{C}}(S)$ which satisfies $f s=f$ is an isomorphism. A minimal left approximation is a left minimal left approximation. Similarly, minimal right approximations are defined. 
In the abelian category $\bmod \Lambda$, a morphism $f: A \rightarrow B$ has a factorization $A \rightarrow$ $\operatorname{Im}(f) \rightarrow B$ over the image. The two maps $f_{1}: A \rightarrow \operatorname{Im}(f)$ and $f_{2}: \operatorname{Im}(f) \rightarrow B$ are determined uniquely as objects in $\mathcal{H}(\Lambda)$, up to isomorphism, and give rise to functors

$$
\begin{array}{clll}
\text { Epi : } & \mathcal{H}(\Lambda) \rightarrow \mathcal{F}(\Lambda), & & (A \stackrel{f}{\rightarrow} B) \mapsto\left(A \stackrel{f_{1}}{\rightarrow} \operatorname{Im}(f)\right), \\
\text { Mono : } & \mathcal{H}(\Lambda) \rightarrow \mathcal{S}(\Lambda), & (A \stackrel{f}{\rightarrow} B) \mapsto\left(\operatorname{Im}(f) \stackrel{f_{2}}{\rightarrow} B\right) .
\end{array}
$$

There are functorial isomorphisms Epi $\cong$ Cok Ker and Mono $\cong$ Ker Cok.

Lemma 2.1 (Approximations given by Mono and Epi). Let $(f: A \rightarrow B)$ be an object in $\mathcal{H}(\Lambda)$.

1. The map $\left(f_{1}, 1_{B}\right): f \rightarrow \operatorname{Mono}(f)$ is a minimal left approximation of $f$ in $\mathcal{S}(\Lambda)$.

2. The map $\left(1_{A}, f_{2}\right): \operatorname{Epi}(f) \rightarrow f$ is a minimal right approximation of $f$ in $\mathcal{F}(\Lambda)$.

The proof is immediate from the definitions.

For the object $(f: A \rightarrow B) \in \mathcal{H}(\Lambda)$ there is also a minimal right approximation in $\mathcal{S}(\Lambda)$ and a minimal left approximation in $\mathcal{F}(\Lambda)$, as we are going to show.

First we define Mimo $(f)$, the minimal monomorphism for $f$, as follows: Let $e^{\prime}: \operatorname{Ker}(f) \rightarrow \mathrm{I} \operatorname{Ker}(f)$ be an injective envelope and choose an extension $e: A \rightarrow$ $\operatorname{IKer}(f)$ of $e^{\prime}$; thus $e^{\prime}=f^{\prime} e$, where $f^{\prime}: \operatorname{Ker}(f) \rightarrow A$ is the inclusion map. Then $\operatorname{Mimo}(f)$ is the map

$$
\operatorname{Mimo}(f)=\left[\begin{array}{ll}
f & e
\end{array}\right]: \quad A \rightarrow B \oplus \operatorname{IKer}(f) .
$$

Lemma 2.2 (Mimo is well-defined). $\operatorname{Mimo}(f)$ is independent of the choice of e, up to isomorphism in $\mathcal{H}(\Lambda)$.

Proof. Let $e_{1}, e_{2}: A \rightarrow \operatorname{IKer}(f)$ be two extensions of $e^{\prime}$, thus $e^{\prime}=f^{\prime} e_{1}=f^{\prime} e_{2}$. We see that the difference $e_{2}-e_{1}$ vanishes on $\operatorname{Ker}(f)$. Write $f=f_{1} f_{2}$ with $f_{1}$ an epimorphism and $f_{2}$ a monomorphism. Since $e_{2}-e_{1}$ vanishes on $\operatorname{Ker}(f)$, it factorizes through $f_{1}=\operatorname{Cok}\left(f^{\prime}\right)$, thus $e_{2}-e_{1}=f_{1} \hat{e}$ for some map $\hat{e}: \operatorname{Im}(f) \rightarrow \operatorname{IKer}(f)$. Since $f_{2}$ is mono and the target $\operatorname{IKer}(f)$ of $\hat{e}$ is injective, we can extend $\hat{e}$ to $B$ : There is $\tilde{e}: B \rightarrow \operatorname{IKer}(f)$ such that $f_{2} \tilde{e}=\hat{e}$. Altogether we have $f \tilde{e}=f_{1} f_{2} \tilde{e}=f_{1} \hat{e}=e_{2}-e_{1}$. It follows that the representations in $\mathcal{S}(\Lambda)$ given by $\left[\begin{array}{ll}f & e_{1}\end{array}\right]$ and by $\left[\begin{array}{ll}f & e_{2}\end{array}\right]$ are isomorphic:

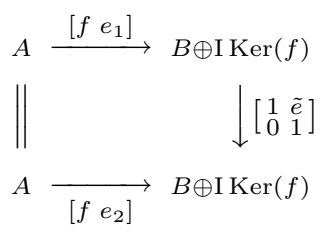

Dually one defines the minimal epimorphism, $\operatorname{Mepi}(f)$, for a map $f: A \rightarrow B$ as follows. The projective cover of the cokernel of $f, p^{\prime}: \operatorname{PCok}(f) \rightarrow \operatorname{Cok}(f)$ factorizes over the canonical map $f^{\prime \prime}: B \rightarrow \operatorname{Cok}(f)$ so there is $p: \operatorname{PCok}(f) \rightarrow B$ such that $p^{\prime}=p f^{\prime \prime}$. Let $\operatorname{Mepi}(f)$ denote the map

$$
\operatorname{Mepi}(f)=\left[\begin{array}{l}
f \\
p
\end{array}\right]: \quad A \oplus \mathrm{P} \operatorname{Cok}(f) \longrightarrow B ;
$$

then the dual version of the above result holds for Mepi. 
Lemma 2.3 (Mepi is well-defined). Mepi $(f)$ is independent of the choice of $p$, up to isomorphism in $\mathcal{H}(\Lambda)$.

There are canonical maps $\operatorname{Mimo}(f) \rightarrow f$ and $f \rightarrow \operatorname{Mepi}(f)$,

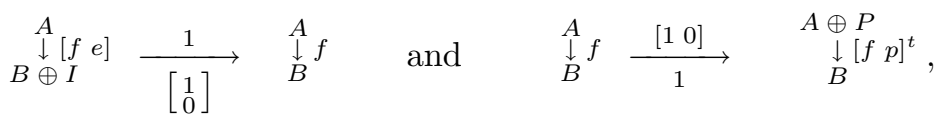

which give rise to a "dual" version of Lemma 2.1:

Proposition 2.4 (Approximations defined by Mimo and Mepi). Let $(f: A \rightarrow B)$ be an object in $\mathcal{H}(\Lambda)$.

1. The map $\operatorname{Mimo}(f) \rightarrow f$ is a minimal right approximation of $f$ in $\mathcal{S}(\Lambda)$.

2. The map $f \rightarrow \operatorname{Mepi}(f)$ is a minimal left approximation of $f$ in $\mathcal{F}(\Lambda)$.

Proof of the first statement. Let $(g: C \rightarrow D)$ be an object in $\mathcal{S}(\Lambda)$ and $(u, v)$ a morphism from $g$ to $f$. We need to find $\left(u^{\prime}, v^{\prime}\right): g \rightarrow \operatorname{Mimo}(f)$ such that the composition with the above map $F: \operatorname{Mimo}(f) \rightarrow f$ is just $(u, v)$. Since $g: C \rightarrow D$ is a monomorphism, the composition $C \stackrel{u}{\rightarrow} A \stackrel{e}{\rightarrow} I$ lifts to a map $v_{2}: D \rightarrow I$ such that $u e=g v_{2}$. Then the pair $\left(u^{\prime}, v^{\prime}\right)=\left(u,\left[v, v_{2}\right]\right)$ is a morphism $g \rightarrow \operatorname{Mimo}(f)$ which satisfies the condition that $\left(u^{\prime}, v^{\prime}\right) F=(u, v)$. Thus, $F$ is a right approximation. It remains to show that $F$ is right minimal. Let $(u, v)$ be an endomorphism of $\operatorname{Mimo}(f)$ such that $F=(u, v) F$ holds and write the map $v: B \oplus I \rightarrow B \oplus I$ as a matrix $v=\left[\begin{array}{ll}v_{11} & v_{12} \\ v_{21} & v_{22}\end{array}\right]$. Since $F=(u, v) F$ holds, it follows that $u=1_{A}, v_{11}=1_{B}$, and $v_{21}=0$. Now the condition that $(u, v)$ is a

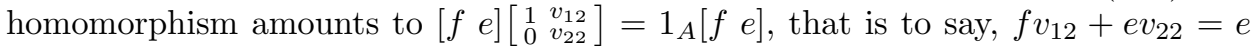
must hold. Restricting both sides to the kernel of $f$ (so that the composition $f v_{12}$ vanishes) yields that the two maps $e v_{22}, e: \operatorname{Ker}(f) \rightarrow I$ are equal. Since $I$ is the injective envelope of $\operatorname{Ker}(f)$, minimality implies that $v_{22}$ is an automorphism of $I$. Hence $v=\left[\begin{array}{cc}1_{B} & v_{12} \\ 0 & v_{22}\end{array}\right]$ is an automorphism of $B \oplus I$. We have shown that $F$ is right minimal.

As a consequence of Lemma 2.1 and Proposition 2.4 we obtain:

Theorem 2.5 (Existence of Auslander-Reiten sequences). The subcategories $\mathcal{S}(\Lambda)$ and $\mathcal{F}(\Lambda)$ are functorially finite in $\mathcal{H}(\Lambda)$ and hence have Auslander-Reiten sequences.

Proof. According to the first statements in Lemma 2.1 and Proposition 2.4, each object $f$ in $\mathcal{H}(\Lambda)$ has a left and a right approximation in $\mathcal{S}(\Lambda)$, this is to say, $\mathcal{S}(\Lambda)$ is functorially finite. Similarly it follows from the second statements in Lemma 2.1 and Proposition 2.4 that the category $\mathcal{F}(\Lambda)$ is functorially finite, too. According to [AS, Theorem 2.4], those categories have Auslander-Reiten sequences.

\section{Transfer of Auslander-Reiten sequences}

In this section we construct Auslander-Reiten sequences for the categories $\mathcal{S}(\Lambda)$ and $\mathcal{F}(\Lambda)$ from corresponding sequences in the module category $\mathcal{H}(\Lambda)$. Surprisingly, the converse is also possible: Auslander-Reiten sequences in $\mathcal{S}(\Lambda)$ and $\mathcal{F}(\Lambda)$ give rise to Auslander-Reiten sequences in $\mathcal{H}(\Lambda)$. First we need a lemma. 
Lemma 3.1 (Kernels and Cokernels of Auslander-Reiten sequences). Suppose the following Auslander-Reiten sequence in the category $\mathcal{H}(\Lambda)$ is given:

$$
0 \longrightarrow \begin{array}{ccccc}
A \\
\downarrow \\
A_{1}
\end{array} \stackrel{f}{f_{1}} \quad \begin{gathered}
B \\
B_{1}
\end{gathered} \underset{g_{1}}{\longrightarrow} \underset{C_{1}}{\downarrow} \longrightarrow 0
$$

If the kernel $\operatorname{Ker}(c)=\left(c^{\prime}: C^{\prime} \rightarrow C\right)$ of the end term is not a projective object, then the sequence obtained by applying the kernel functor,

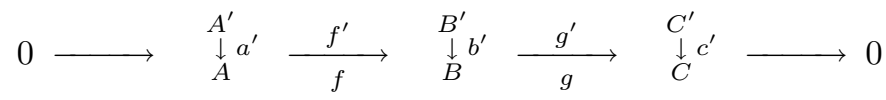

is either split exact or almost split in $\mathcal{S}(\Lambda)$. Dually, if the cokernel

$$
\operatorname{Cok}(a)=\left(a^{\prime \prime}: A_{1} \rightarrow A_{1}^{\prime \prime}\right)
$$

of the first term is not an injective object, then the sequence obtained by using the cokernel functor,

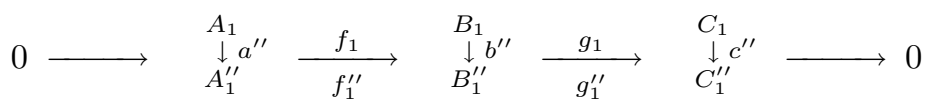

is either split exact or almost split in $\mathcal{F}(\Lambda)$.

Proof. We only show the statement about the sequence in $\mathcal{S}(\Lambda)$, and for this sequence we only show that the map $\left(g^{\prime}, g\right):\left(b^{\prime}: B^{\prime} \rightarrow B\right) \rightarrow\left(c^{\prime}: C^{\prime} \rightarrow C\right)$ is either a split epimorphism or a right almost split morphism. Note that this implies that $g^{\prime}$ is onto.

Let $\left(x^{\prime}: X^{\prime} \rightarrow X\right)$ be an object in $\mathcal{S}(\Lambda)$ and $\left(t^{\prime}, t\right): x^{\prime} \rightarrow c^{\prime}$ a morphism which is not a split epimorphism. Let $x: X \rightarrow X_{1}$ be the cokernel for $x^{\prime}$, factorize $c=c_{1} c_{2}$ as the product of an epimorphism $c_{1}: C \rightarrow \operatorname{Im}(c)$ and a monomorphism $c_{2}: \operatorname{Im}(c) \rightarrow C_{1}$, and let $t_{1}: X_{1} \rightarrow \operatorname{Im}(c)$ be the cokernel map for $\left(t^{\prime}, t\right)$; this map satisfies $x t_{1}=t c_{1}$. Then $\left(t, t_{1} c_{2}\right): x \rightarrow c$ is not a split epimorphism, since its kernel is not, and hence factorizes over the map $\left(g, g_{1}\right)$ : There is

$$
\left(u, u_{1}\right):\left(x: X \rightarrow X_{1}\right) \rightarrow\left(b: B \rightarrow B_{1}\right)
$$

such that $\left(t, t_{1} c_{2}\right)=\left(u, u_{1}\right)\left(g, g_{1}\right)$. If $u^{\prime}: X^{\prime} \rightarrow B^{\prime}$ is the kernel map for $\left(u, u_{1}\right)$, then $\left(t^{\prime}, t\right)=\left(u^{\prime}, u\right)\left(g^{\prime}, g\right)$ factorizes over $\left(g^{\prime}, g\right)$.

Proposition 3.2 (Transfer of AR sequences from $\mathcal{H}(\Lambda)$ to $\mathcal{S}(\Lambda)$ and $\mathcal{F}(\Lambda)$ ). Suppose that $0 \rightarrow a \rightarrow b \rightarrow c \rightarrow 0$ is an Auslander-Reiten sequence in $\mathcal{H}(\Lambda)$.

1. If $c$ is an indecomposable nonprojective object in $\mathcal{F}(\Lambda)$, then

$$
0 \rightarrow \operatorname{Epi}(a) \rightarrow \operatorname{Epi}(b) \rightarrow c \rightarrow 0
$$

is an Auslander-Reiten sequence in $\mathcal{F}(\Lambda)$ and

$$
0 \rightarrow \operatorname{Ker}(a) \rightarrow \operatorname{Ker}(b) \rightarrow \operatorname{Ker}(c) \rightarrow 0
$$

is an Auslander-Reiten sequence in $\mathcal{S}(\Lambda)$.

2. If $a$ is an indecomposable noninjective object in $\mathcal{S}(\Lambda)$, then

$$
0 \rightarrow a \rightarrow \operatorname{Mono}(b) \rightarrow \operatorname{Mono}(c) \rightarrow 0
$$

is an Auslander-Reiten sequence in $\mathcal{S}(\Lambda)$ and

$$
0 \rightarrow \operatorname{Cok}(a) \rightarrow \operatorname{Cok}(b) \rightarrow \operatorname{Cok}(c) \rightarrow 0
$$

is an Auslander-Reiten sequence in $\mathcal{F}(\Lambda)$. 
Proof. We show the first assertion. Note that the sequence $0 \rightarrow \operatorname{Epi}(a) \rightarrow \operatorname{Epi}(b) \rightarrow$ $c \rightarrow 0$ in $\mathcal{F}(\Lambda)$ is the cokernel sequence for $0 \rightarrow \operatorname{Ker}(a) \rightarrow \operatorname{Ker}(b) \rightarrow \operatorname{Ker}(c) \rightarrow 0$ in $\mathcal{S}(\Lambda)$ since $c \in \mathcal{F}(\Lambda)$ satisfies $c=\operatorname{Epi}(c)=\operatorname{Cok} \operatorname{Ker}(c)$. By Lemma 3.1, both sequences are either split exact or almost split in their respective categories. The sequences are not split exact since the morphism $\operatorname{Epi}(b) \rightarrow c$ is the composition of the right approximation $\operatorname{Epi}(b) \rightarrow b$ and the right almost split morphism $b \rightarrow c$, and hence is a right almost split morphism.

Corollary 3.3 (The translation in $\mathcal{H}(\Lambda), \mathcal{S}(\Lambda)$ and $\mathcal{F}(\Lambda)$ ).

1. If $c \in \mathcal{F}(\Lambda)$ is indecomposable nonprojective, then $\tau_{\mathcal{F}}(c)=\operatorname{Epi} \tau_{\mathcal{H}}(c)$.

2. If $c \in \mathcal{S}(\Lambda)$ is indecomposable nonprojective, then $\tau_{\mathcal{S}}(c)=\operatorname{Ker} \tau_{\mathcal{H}} \operatorname{Cok}(c)$.

3. If $a \in \mathcal{S}(\Lambda)$ is indecomposable noninjective, then $\tau_{\mathcal{S}}^{-}(a)=\operatorname{Mono} \tau_{\mathcal{H}}^{-}(a)$.

4. If $a \in \mathcal{F}(\Lambda)$ is indecomposable noninjective, then $\tau_{\mathcal{F}}^{-}(a)=\operatorname{Cok} \tau_{\mathcal{H}}^{-} \operatorname{Ker}(a)$.

In the remainder of this section we describe how Auslander-Reiten sequences in $\mathcal{S}(\Lambda)$ and $\mathcal{F}(\Lambda)$ give rise to Auslander-Reiten sequences in $\mathcal{H}(\Lambda)$.

Lemma 3.4 (The functors Epi and Mono reflect some split morphisms). Let $(f, g)$ : $(A \stackrel{a}{\rightarrow} B) \rightarrow(C \stackrel{c}{\rightarrow} D)$ be a morphism in $\mathcal{H}(\Lambda)$ and let $h: \operatorname{Im}(a) \rightarrow \operatorname{Im}(c)$ be the induced map on the images:

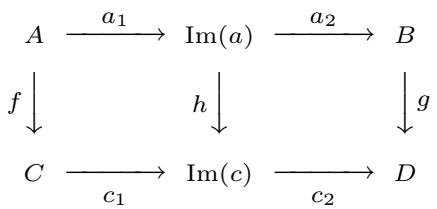

1. If $(f, g)$ is split monic (split epic), then $\operatorname{Epi}(f, g)=(f, h)$ and $\operatorname{Mono}(f, g)=$ $(h, g)$ are both split monic (split epic).

2. If $a_{2}: \operatorname{Im}(a) \rightarrow B$ is an injective envelope, and if $\operatorname{Epi}(f, g)$ is split monic, then so is $(f, g)$.

3. If $c_{1}: C \rightarrow \operatorname{Im}(c)$ is a projective cover, and if $\operatorname{Mono}(f, g)$ is split epic, then so is $(f, g)$.

Proof. The first assertion is clear since Epi and Mono are functors; the third assertion is dual to the second, so we only consider the second assertion. Suppose that $(f, h)$ is split monic, so there are maps $u: C \rightarrow A$ and $w: \operatorname{Im}(c) \rightarrow \operatorname{Im}(a)$ such that $u a_{1}=c_{1} w, f u=1_{A}, h w=1_{\operatorname{Im}(a)}$. Since $B$ is injective, the map $w a_{2}$ extends to $D$ : There is $v: D \rightarrow B$ such that $c_{2} v=w a_{2}$. Since $a_{2} g v=h c_{2} v=h w a_{2}=a_{2}$, the left minimality of the injective envelope $a_{2}$ implies that $g v$ is an automorphism of $B$. It follows that the morphism $(f, g)$ is split monic.

Proposition 3.5 (Transfer of AR sequences from $\mathcal{S}(\Lambda)$ and $\mathcal{F}(\Lambda)$ to $\mathcal{H}(\Lambda)$ ).

1. Let $\left(c: C \rightarrow C^{\prime \prime}\right)$ be an indecomposable nonprojective object in $\mathcal{F}(\Lambda)$. If the first two rows in the diagram

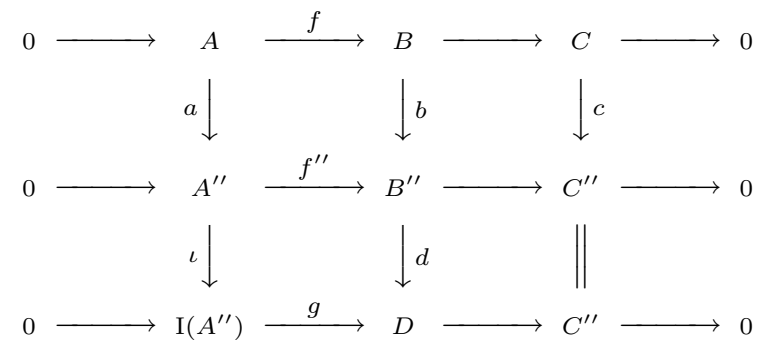


define an Auslander-Reiten sequence in $\mathcal{F}(\Lambda)$ and if the third row is obtained as a push-out along the injective envelope $\iota: A^{\prime \prime} \rightarrow \mathrm{I}\left(A^{\prime \prime}\right)$, then the first row and the third row define an Auslander-Reiten sequence in $\mathcal{H}(\Lambda)$.

2. Let $\left(a: A^{\prime} \rightarrow A\right)$ be an indecomposable noninjective object in $\mathcal{S}(\Lambda)$. If the two lower rows in the diagram

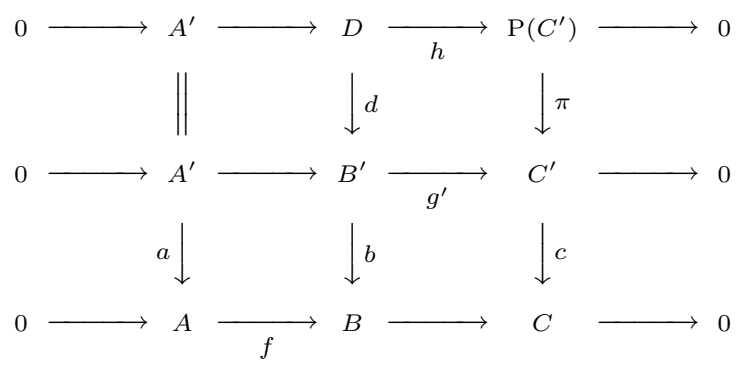

define an Auslander-Reiten sequence in $\mathcal{S}(\Lambda)$ and if the first row is obtained as pull-back along the projective cover $\pi: \mathrm{P}\left(C^{\prime}\right) \rightarrow C^{\prime}$, then the first row and the third row define an Auslander-Reiten sequence in $\mathcal{H}(\Lambda)$.

Proof of of the first statement. The map $(f, g)$ in $\mathcal{H}(\Lambda)$ is not a split monomorphism since $\operatorname{Epi}(f, g)=\left(f, f^{\prime \prime}\right)$ is not, by Lemma 3.1. We show that $(f, g)$ is left almost split. Suppose

$$
(r, s): \quad\left(A \stackrel{a \iota}{\longrightarrow} \mathrm{I}\left(A^{\prime \prime}\right)\right) \quad \longrightarrow \quad(X \stackrel{x}{\rightarrow} Y)
$$

is a morphism in $\mathcal{H}(\Lambda)$ which is not a split monomorphism. Factorize $x$ over the image as $X \stackrel{x_{1}}{\rightarrow} \operatorname{Im}(x) \stackrel{x_{2}}{\rightarrow} Y$, so $x=x_{1} x_{2}$. Then there is $t: A^{\prime \prime} \rightarrow \operatorname{Im}(x)$ such that the two squares commute:

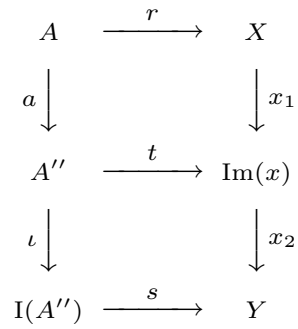

By Lemma 3.4, part 2, the morphism $(r, t)$ is not split monic in $\mathcal{F}(\Lambda)$ and hence factorizes over $\left(f, f^{\prime \prime}\right)$ : There are $v: B \rightarrow X, v^{\prime \prime}: B^{\prime \prime} \rightarrow \operatorname{Im}(x)$ such that

$$
b v^{\prime \prime}=v x_{1}, \quad \text { and } \quad(r, t)=\left(f, f^{\prime \prime}\right)\left(v, v^{\prime \prime}\right) .
$$

Then $f^{\prime \prime}\left(v^{\prime \prime} x_{2}\right)=t x_{2}=\iota s$, so we obtain $w: D \rightarrow Y$ such that $d w=v^{\prime \prime} x_{2}$ and $g w=s$, by the push-out property for $Y$. Thus, $(v, w)$ is a morphism in $\mathcal{H}(\Lambda)$ and our test map $(r, s)$ factorizes over $(f, g):(r, s)=(f, g)(v, w)$. For the proof that $(f, g)$ is a source map in the module category $\mathcal{H}(\Lambda)$, it remains to check that $(f, g)$ is left minimal, and this follows from the indecomposability of the cokernel $\left(C \rightarrow C^{\prime \prime}\right)$.

\section{Minimal monomorphisms and the stable CATEgory}

Returning to the investigation of minimal monomorphisms, we show that if $f, g$ : $A \rightarrow B$ are two maps which differ by a morphism which factorizes through an injective module, then $\operatorname{Mimo}(f)$ and $\operatorname{Mimo}(g)$ are isomorphic as objects in $\mathcal{S}(\Lambda)$. 
First we verify three claims.

Claim 1. Let $f, g: A \rightarrow B$ be maps in $\bmod \Lambda$ such that $g-f$ factorizes through an injective module. Let $h: A \rightarrow \mathrm{I}(A)$ be an injective envelope. Then the objects in $\mathcal{S}(\Lambda)$ given by the monomorphisms $[f h]$ and $[g h]$ are isomorphic.

Proof. The map $g-f$ factorizes through the injective envelope $h$, so there is $u$ : $\mathrm{I}(A) \rightarrow B$ such that $g-f=h u$. The following commutative diagram shows that $[f h]$ and $[g h]$ are isomorphic objects in $\mathcal{H}(\Lambda)$ :

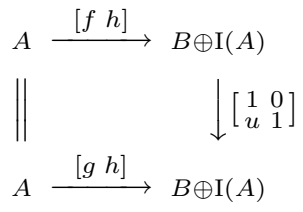

Claim 2. Given $f: A \rightarrow B$ and a map $h: A \rightarrow I$ with $I$ injective such that $[f h]: A \rightarrow B \oplus I$ is a monomorphism, then there is an injective module $J$ and a commutative diagram

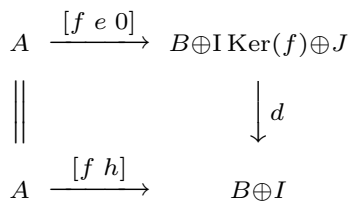

with $d$ an isomorphism and $e: A \rightarrow \operatorname{IKer}(f)$ an extension of the injective envelope $\operatorname{Ker}(f) \rightarrow \operatorname{IKer}(f)$.

The diagram shows that the maps given by the two rows are isomorphic as objects in $\mathcal{H}(\Lambda)$. Note that in $\mathcal{H}(\Lambda)$, the object given by the upper row decomposes as the direct sum of the two objects $([f e]: A \rightarrow B \oplus \operatorname{IKer}(f))$ and $(0 \rightarrow J)$. Up to isomorphism, the first one is just $\operatorname{Mimo}(f)$.

Proof. Since $[f h]$ is a monomorphism, the restriction of $h$ to $\operatorname{Ker}(f)$ is injective. Thus $I$ contains a submodule isomorphic to $\operatorname{Ker}(f)$ and therefore $I$ decomposes as $I=\operatorname{IKer}(f) \oplus J$ for some injective $\Lambda$-module $J$. Then $h$ has the form $h=\left[e h_{2}\right]$, where $e: A \rightarrow \operatorname{IKer}(f)$ is an extension of the inclusion $\operatorname{Ker}(f) \rightarrow \operatorname{IKer}(f)$, and $h_{2}: A \rightarrow J$ satisfies $h_{2} \operatorname{Ker}(f)=0$. Write $f=f_{1} f_{2}$ with $f_{1}$ an epimorphism and $f_{2}$ a monomorphism. Since $h_{2}$ vanishes on $\operatorname{Ker}(f)$, we can factorize $h_{2}$ through $f_{1}$, say $h_{2}=f_{1} v$ for some map $v: \operatorname{Im}(f) \rightarrow J$. Since $J$ is injective and $f_{2}$ a monomorphism, we obtain a lifting $w: B \rightarrow J$ of $v$ to $B$, thus $v=f_{2} w$. Altogether we see that $h_{2}=f_{1} v=f_{1} f_{2} w=f w$. This shows that the following diagram commutes:

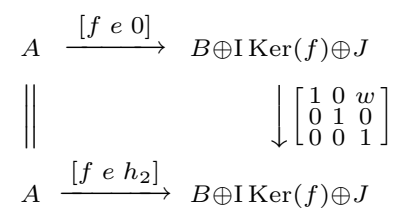

Claim 3. Suppose that $B$ has no nonzero injective direct summands and that $f, g$ : $A \rightarrow B$ are maps such that $g-f$ factorizes through an injective object. Then $\operatorname{IKer}(f)=\operatorname{IKer}(g)$. 
Proof. (a) First we show that if a map $h: A \rightarrow B$ factorizes through an injective object, say $h=h_{1} h_{2}$ where $h_{1}: A \rightarrow I$ and $h_{2}: I \rightarrow B$, then $\operatorname{Soc} \operatorname{Ker}(h)=\operatorname{Soc}(A)$. Indeed, if there were a simple submodule $S$ of $A$ such that the composition

$$
S \stackrel{\text { incl }}{\longrightarrow} A \stackrel{h_{1}}{\longrightarrow} I \stackrel{h_{2}}{\longrightarrow} B
$$

is nonzero, then we would obtain that $B$ has a nonzero injective direct summand - in contradiction to our assumption on $B$.

(b) As a consequence we obtain that if $g-f$ factorizes through an injective object, then $\operatorname{Soc} \operatorname{Ker}(f)=\operatorname{Soc} \operatorname{Ker}(g)$ holds. By (a), $\operatorname{Soc}(A) \subseteq \operatorname{Ker}(g-f)$ and hence

$$
\operatorname{Ker}(f) \cap \operatorname{Soc}(A)=\operatorname{Ker}(f+(g-f)) \cap \operatorname{Soc}(A)=\operatorname{Ker}(g) \cap \operatorname{Soc}(A) .
$$

Thus, I $\operatorname{Ker}(f)=\mathrm{I} \operatorname{Soc} \operatorname{Ker}(f)=\mathrm{I} \operatorname{Soc} \operatorname{Ker}(g)=\mathrm{I} \operatorname{Ker}(g)$ holds.

Proposition 4.1 (Mimo independent of maps which factorize through injective modules). Suppose that $B$ has no nonzero injective direct summands. Let $f, g$ : $A \rightarrow B$ be maps in $\bmod \Lambda$ such that $g-f$ factorizes through an injective $\Lambda$-module. Then the objects $\operatorname{Mimo}(f), \operatorname{Mimo}(g)$ are isomorphic in $\mathcal{S}(\Lambda)$.

Proof. Let $h: A \rightarrow \mathrm{I}(A)$ be an injective envelope; then the objects $[f h],\left[\begin{array}{ll}g & h\end{array}\right]$ are isomorphic by Claim 1. According to Claim 2, there exist injective modules $J_{1}, J_{2}$, extensions $e_{1}: A \rightarrow \operatorname{IKer}(f), e_{2}: A \rightarrow \operatorname{IKer}(g)$ of the inclusion maps $\operatorname{Ker}(f) \rightarrow \operatorname{IKer}(f), \operatorname{Ker}(g) \rightarrow \operatorname{IKer}(g)$, respectively, and isomorphisms $d_{1}, d_{2}$ such that the diagram below is commutative:

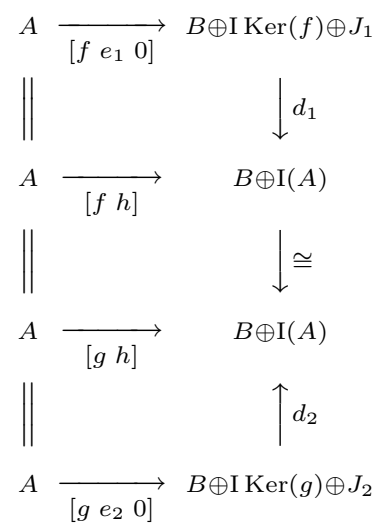

According to Claim 3, the $\Lambda$-modules $\operatorname{IKer}(f), \operatorname{IKer}(g)$ are isomorphic. By the Krull-Remak-Schmidt theorem for $\Lambda$-modules, $J_{1} \cong J_{2}$ follows. Note that the top row in the diagram when considered as an object in $\mathcal{H}(\Lambda)$ is isomorphic to the direct $\operatorname{sum} \operatorname{Mimo}(f) \oplus\left(0 \rightarrow J_{1}\right)$, while the bottom row is isomorphic to $\operatorname{Mimo}(g) \oplus(0 \rightarrow$ $\left.J_{2}\right)$. Applying the Krull-Remak-Schmidt theorem in the category $\mathcal{H}(\Lambda)$ we obtain that $\operatorname{Mimo}(f) \cong \operatorname{Mimo}(g)$.

The following example shows that the condition that $B$ has no nonzero injective direct summands cannot be omitted.

Example. Let $\Lambda$ be a uniserial ring of Loewy length 2 with $m$ a generator of the maximal ideal $\mathbf{m}$. Denote by $\mu_{m}: \Lambda \rightarrow \Lambda$ the multiplication by $m$. With $f=1: \Lambda \rightarrow \Lambda$ and $g=\mu_{m}: \Lambda \rightarrow \Lambda$, clearly $g-f$ factorizes through an injective 
$\Lambda$-module, but $\operatorname{Mimo}(f)=f$ and $\operatorname{Mimo}(g)=\left(\Lambda \stackrel{\left[\mu_{2} 1\right]}{\longrightarrow} \Lambda \oplus \Lambda\right)$ are not isomorphic as $\operatorname{Mimo}(g) \cong f \oplus(0 \rightarrow \Lambda)$.

The criterion in Proposition 4.1 for $\operatorname{Mimo}(f) \cong \operatorname{Mimo}(g)$ can be refined to obtain an equivalent condition.

Theorem 4.2 (An equivalent condition for $\operatorname{Mimo}(f) \cong \operatorname{Mimo}(g)$ ). Let $f, g: A \rightarrow$ $B$ be maps in $\bmod \Lambda$ and suppose that $B$ has no nonzero injective direct summands. Then $\operatorname{Mimo}(f)$ and $\operatorname{Mimo}(g)$ are isomorphic in $\mathcal{S}(\Lambda)$ if and only if there are automorphisms a of $A$ and $b$ of $B$ such that $f b-a g$ factorizes through an injective $\Lambda$-module.

Proof. Assume first that $\operatorname{Mimo}(f): A \stackrel{\left[f e_{1}\right]}{\longrightarrow} B \oplus I$ and $\operatorname{Mimo}(g): A \stackrel{\left[g e_{2}\right]}{\longrightarrow} B \oplus I$ are isomorphic. Then there are maps $a \in \operatorname{Aut} A$ and $h=\left(\begin{array}{cc}b & h_{21} \\ h_{12} & h_{22}\end{array}\right) \in \operatorname{Aut}(B \oplus I)$ such that $\left[f e_{1}\right] h=a\left[g e_{2}\right]$. Thus, $a g=f b+e_{1} h_{12}$, so $a g-f b$ factorizes through the injective $\Lambda$-module $I$. Moreover, as $B$ and $I$ have no indecomposable direct summands in common, it follows that the map $h$ is an automorphism if and only if both $b$ and $h_{22}$ are automorphisms.

For the converse, assume that $f b-a g$ factorizes through an injective $\Lambda$-module. By Proposition 4.1, the objects $\operatorname{Mimo}(f b)$ and $\operatorname{Mimo}(a g)$ are isomorphic; assume they are given by maps

$$
\operatorname{Mimo}(f b)=\left[\begin{array}{ll}
f b & e_{1}
\end{array}\right], \operatorname{Mimo}(a g)=\left[\begin{array}{ll}
a g & e_{2}
\end{array}\right]: \quad A \longrightarrow B \oplus I .
$$

Thus, $\operatorname{Mimo}(f) \cong \operatorname{Mimo}(g)$, as indicated by the following diagram:

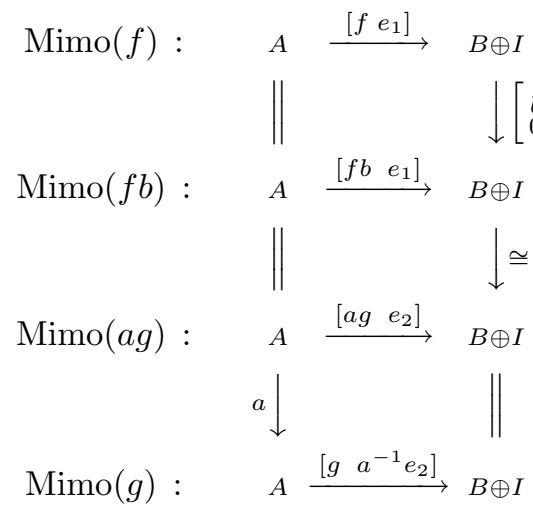

For the last step note that if $e_{2}: A \rightarrow I$ is an extension to $A$ of an injective envelope for $\operatorname{Ker}(a g)$, then since $\operatorname{Ker}(a g)=a^{-1} \operatorname{Ker}(g)$, one obtains that $a^{-1} e_{2}$ is an extension to $A$ of an injective envelope for $\operatorname{Ker}(g)$. By Lemma 2.2, the object ( $\left.\left[g a^{-1} e_{2}\right]: A \rightarrow B \oplus I\right)$ is $\operatorname{Mimo}(g)$, up to isomorphism.

There is the following dual result for minimal epimorphisms.

Theorem 4.3 (An equivalent condition for Mepi $(f) \cong \operatorname{Mepi}(g)$ ). Let $f, g: A \rightarrow B$ be maps in $\bmod \Lambda$ and suppose that $A$ has no nonzero projective direct summands. Then $\operatorname{Mepi}(f)$ and $\operatorname{Mepi}(g)$ are isomorphic in $\mathcal{F}(\Lambda)$ if and only if there are automorphisms a of $A$ and $b$ of $B$ such that $f b-a g$ factorizes through a projective $\Lambda$-module. 


\section{The Auslander-Reiten translation}

In Chapter 3 we have seen how the Auslander-Reiten translations in the categories $\mathcal{H}(\Lambda), \mathcal{S}(\Lambda)$, and $\mathcal{F}(\Lambda)$ are related. Using this, we develop a formula to compute the Auslander-Reiten translate $\tau_{\mathcal{S}}(f)$ of an object $(f: A \rightarrow B)$ in $\mathcal{S}(\Lambda)$ directly in the category $\bmod \Lambda$. Here is the main result:

Theorem 5.1 (The Auslander-Reiten translation in $\mathcal{S}(\Lambda)$ ). For an object $(A \stackrel{f}{\rightarrow} B)$ in $\mathcal{S}(\Lambda)$, the Auslander-Reiten translate is given by

$$
\tau_{\mathcal{S}}(f)=\operatorname{Mimo} \tau_{\Lambda} \operatorname{Cok}(f) .
$$

Note that this means the following: We start with the monomorphism $f$ and form its cokernel $g$. We apply $\tau_{\Lambda}$ to this map. Recall that $\tau_{\Lambda}(g)$ is only well-defined in the category $\overline{\bmod } \Lambda$ (obtained from $\bmod \Lambda$ by factoring out all the maps which factorize through an injective object). Represent $\tau_{\Lambda}(g)$ by a morphism $h: D \rightarrow E$ in $\bmod \Lambda$ such that $D$ and $E$ have no nonzero injective direct summands. Now apply Mimo. As we have seen in Chapter 4, this yields a well-defined object in $\mathcal{S}(\Lambda)$, up to isomorphism.

Proof. We proceed as follows. Let $(f: A \rightarrow B)$ be an object in $\mathcal{S}(\Lambda)$ with cokernel $(g: B \rightarrow C)$. In Step 1 we obtain an exact sequence defining $\tau_{\Lambda}(g)$; in Step 2 we construct an exact sequence involving $\tau_{\mathcal{H}}(g)$, from which $\tau_{\mathcal{S}}(f)$ is computed as $\operatorname{Ker} \tau_{\mathcal{H}}(g)$ by Corollary 3.3. The formula for the computation of a kernel in Step 3 is used in Step 4 to relate the two exact sequences, and finally, in Step 5, we obtain our result.

Step 1. For an epimorphism $g: B \rightarrow C$ in $\bmod \Lambda$ we determine $\tau_{\Lambda}(g)$. We start with a minimal projective presentation of $B$, say

$$
Q \stackrel{d}{\longrightarrow} P \stackrel{e}{\longrightarrow} B \longrightarrow 0
$$

and a projective presentation of $C$ using the map eg $: P \rightarrow C$ and a projective cover $t: R \rightarrow \operatorname{Ker}(e g)$ to obtain the following commutative diagram with exact rows:

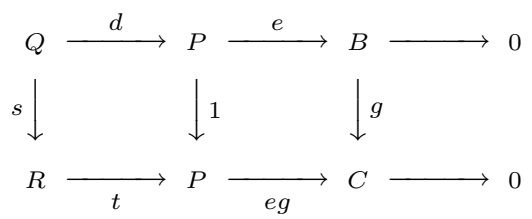

By applying the Nakayama functor $\nu_{\Lambda}=\nu=D \operatorname{Hom}_{\Lambda}\left(-,{ }_{\Lambda} \Lambda\right)$ in $\bmod \Lambda$ to the left square we arrive at the diagram defining $\tau_{\Lambda}(g)$ :

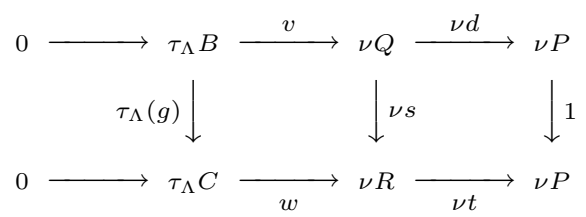

(Since $e g$ is not necessarily a projective cover, $\nu t$ is not necessarily an injective envelope.)

Step 2. From a projective presentation for $g$ in the category $\mathcal{H}(\Lambda)$ we construct an exact sequence which involves $\tau_{\mathcal{H}}(g)$ : Being an epimorphism, the object $g$ has 
a projective cover given by $\left(1_{P}: P \rightarrow P\right)$ with $P$ as in Step 1 . Using the maps $d$ and $t$ we obtain the following projective presentation for $g$ in $\mathcal{H}(\Lambda)$ :

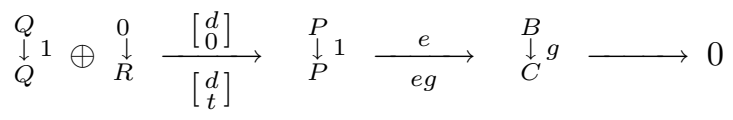

Note that this differs in general from a minimal projective presentation; in order to obtain a minimal one, we would have to split off a direct summand of the form $(0 \rightarrow S)$ where $S$ is a direct summand of $R$ (and $S$ is also a direct summand of $Q$ ). Applying the Nakayama functor $\nu_{\mathcal{H}}$ (see Lemma 1.3-N) to the morphism between the projective modules, we obtain the following sequence:

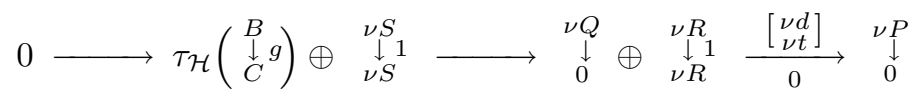

in which the additional projective summand $(0 \rightarrow S)$ gives rise to the injective direct summand $\left(1_{\nu S}: \nu S \rightarrow \nu S\right)$. if

Step 3. For the computation of the kernel of a map $u: U \rightarrow V$ we observe that

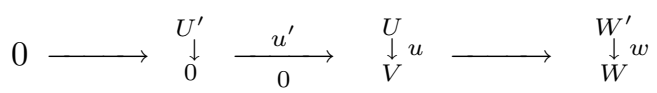

is an exact sequence in $\mathcal{H}(\Lambda)$ with $\left(w: W^{\prime} \rightarrow W\right)$ in $\mathcal{S}(\Lambda)$, then Ker $u=u^{\prime}$.

Step 4 . In the following diagram, the right two columns are split exact and form a commutative diagram in $\mathcal{H}(\Lambda)$; the left column is just the kernel sequence. Note that the sequence in Step 2 involving $\tau_{\mathcal{H}}(g)$ occurs as the middle row, while the sequence in Step 1 defining $\tau_{\Lambda} B$ occurs as the sequence of source modules in the top row.

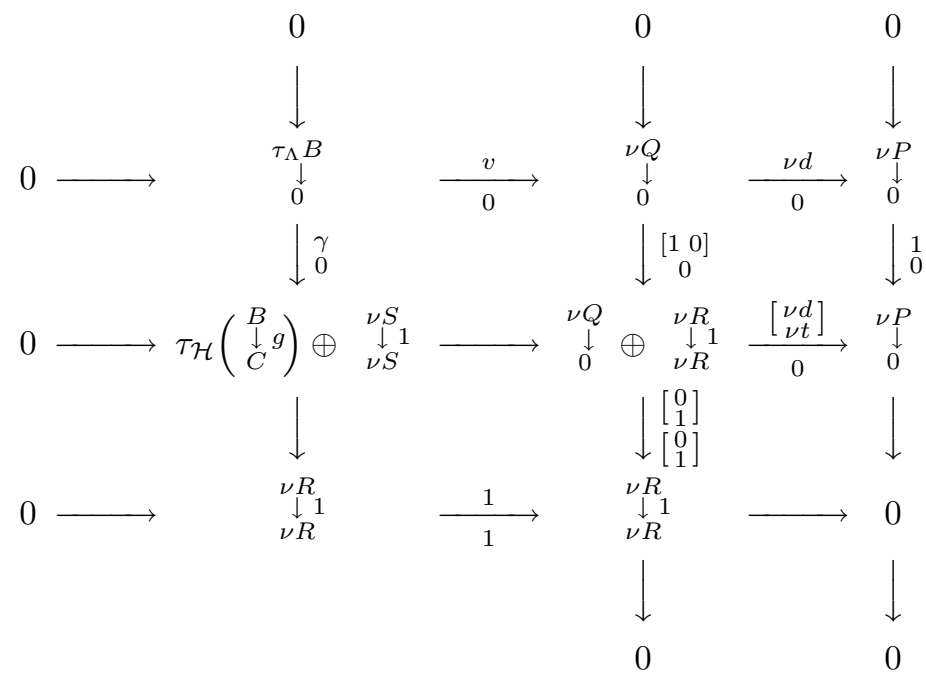

Since the object $1_{\nu R}: \nu R \rightarrow \nu R$ is in $\mathcal{S}(\Lambda)$, we can use the left hand column for the computation of $\operatorname{Ker} \tau_{\mathcal{H}}(g)$, using Step 3:

$$
\operatorname{Ker}\left(\tau_{\mathcal{H}}(g) \oplus\left(1_{\nu S}: \nu S \rightarrow \nu S\right)\right)=\gamma
$$


In order to identify $\gamma$, only the first entries in the above diagram play a role, thus we have to deal with the following diagram:

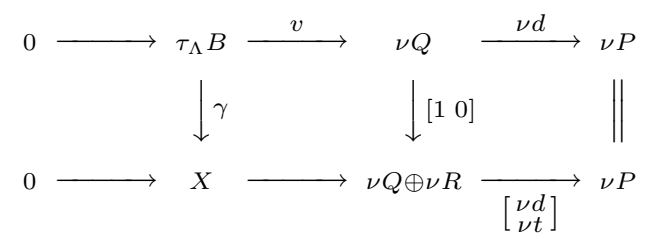

where $X$ is the first component of $\tau_{\mathcal{H}}(g) \oplus\left(1_{\nu S}: \nu S \rightarrow \nu S\right)$. Compare this with the following diagram:

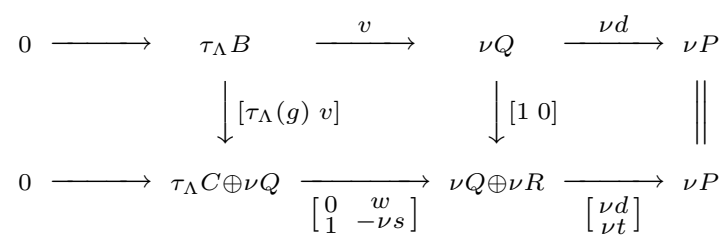

A glance back to Step 1 shows that the diagram is commutative and has an exact upper row. It only has to be confirmed that the lower row is exact. Clearly, the $2 \times 2$-matrix is a monomorphism, and the composition of the last two maps is zero. Now, given $(x, y) \in \nu Q \oplus \nu R$ with $(x) \nu d+(y) \nu t=0$, then $\nu d=\nu s \nu t$ yields that $((x) \nu s+y) \nu t=0$, therefore $(x) \nu s+y$ is in the image of $w$ : There is $z \in \tau_{\Lambda} C$ such that $(z) w=(x) \nu s+y$ and hence

$$
(z, x)\left[\begin{array}{cc}
0 & w \\
1 & -\nu s
\end{array}\right]=(x,(z) w-(x) \nu s)=(x, y) .
$$

Comparing the two diagrams labelled $(*)$ and $(* *)$ we obtain the following isomorphism:

$$
\begin{array}{cc}
\tau_{\Lambda} B \\
\downarrow \gamma \\
X
\end{array} \cong \quad \begin{gathered}
\tau_{\Lambda} B \\
\downarrow\left[\tau_{\Lambda}(g) v\right] \\
\tau_{\Lambda} C \oplus \nu Q
\end{gathered}
$$

Step 5. Write $\tau_{\mathcal{H}}(g)=(h: E \rightarrow F)$, so $\tau_{\mathcal{S}}(f)=\operatorname{Ker} \tau_{\mathcal{H}}(g)=(j: D \rightarrow E)$ where $j=\operatorname{Ker}(h)$. Thus the object given by $\gamma=\operatorname{Ker}\left(\tau_{\mathcal{H}}(g) \oplus\left(1_{\nu S}: \nu S \rightarrow \nu S\right)\right)$ in the previous step has the form

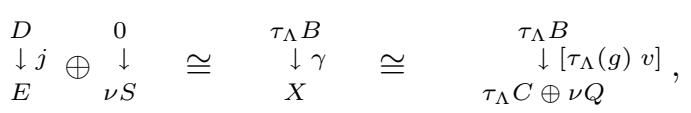

where the second isomorphism has been established in Step 4. By Claim 2 in the previous section, the right hand side is isomorphic to Mimo $\tau_{\Lambda}(g)$, up to an injective direct summand. Since neither $j$ nor Mimo $\tau_{\Lambda}(g)$ has an injective direct summand - recall that $j$ is a $\tau_{\mathcal{S}}$-translate — the Krull-Remak-Schmidt theorem in $\mathcal{S}(\Lambda)$ implies that $\tau_{\mathcal{S}}(f)=j=\operatorname{Mimo} \tau_{\Lambda}(g)$.

There is also the following dual version.

Theorem 5.2 (The Auslander-Reiten translation in $\mathcal{F}(\Lambda))$. For $(A \stackrel{g}{\rightarrow} B)$ an object in $\mathcal{F}(\Lambda)$ the Auslander-Reiten translate is given as

$$
\tau_{\mathcal{F}}^{-}(g)=\operatorname{Mepi} \tau_{\Lambda}^{-} \operatorname{Ker}(g) .
$$


Example. Let $\Lambda$ be a uniserial ring with maximal ideal $\mathbf{m}$, Loewy length $n$ and socle $k=\mathbf{m}^{n-1}$, as in the Example in Chapter 1. Recall that the projective-injective indecomposable $(0 \rightarrow \Lambda)$ in the category $\mathcal{S}(\Lambda)$ has as a sink map the inclusion $(0 \rightarrow \mathbf{m}) \rightarrow(0 \rightarrow \Lambda)$ and as a source map the inclusion $(0 \rightarrow \Lambda) \rightarrow(k \rightarrow \Lambda)$, so $\tau_{\mathcal{S}}(k \rightarrow \Lambda)=(0 \rightarrow \mathbf{m})$. We illustrate the formula in Theorem 5.1 by computing the powers of the Auslander-Reiten translation for the module $(k \rightarrow \Lambda)$. First,

$$
\tau_{\mathcal{S}}\left(\begin{array}{c}
k \\
\downarrow \\
\Lambda
\end{array}\right)=\operatorname{Mimo} \tau_{\Lambda} \operatorname{Cok}\left(\begin{array}{c}
k \\
\downarrow \\
\Lambda
\end{array}\right)=\operatorname{Mimo} \tau_{\Lambda}\left(\begin{array}{c}
\Lambda \\
\downarrow \\
\Lambda / k
\end{array}\right)=\operatorname{Mimo}\left(\begin{array}{c}
0 \\
\downarrow \\
\Lambda / k
\end{array}\right)=\left(\begin{array}{c}
0 \\
\downarrow \\
\Lambda / k
\end{array}\right) \cong\left(\begin{array}{c}
0 \\
\downarrow \\
\mathbf{m}
\end{array}\right)
$$

confirming the above result. Further translates are computed easily:

$$
\begin{aligned}
& \tau_{\mathcal{S}}\left(\begin{array}{c}
0 \\
\downarrow \\
\mathbf{m}
\end{array}\right)=\operatorname{Mimo} \tau_{\Lambda} \operatorname{Cok}\left(\begin{array}{c}
0 \\
\downarrow \\
\mathbf{m}
\end{array}\right)=\operatorname{Mimo} \tau_{\Lambda}\left(\begin{array}{c}
\mathbf{m} \\
\downarrow \\
\mathbf{m}
\end{array}\right)=\left(\begin{array}{c}
\mathbf{m} \\
\downarrow \\
\mathbf{m}
\end{array}\right), \\
& \tau_{\mathcal{S}}\left(\begin{array}{c}
\mathbf{m} \\
\downarrow \\
\mathbf{m}
\end{array}\right)=\operatorname{Mimo} \tau_{\Lambda}\left(\begin{array}{c}
\mathbf{m} \\
\downarrow \\
0
\end{array}\right)=\operatorname{Mimo}\left(\begin{array}{c}
\mathbf{m} \\
\downarrow \\
0
\end{array}\right)=\left(\begin{array}{c}
\mathbf{m} \\
\downarrow \\
\Lambda
\end{array}\right), \\
& \tau_{\mathcal{S}}\left(\begin{array}{c}
\mathbf{m} \\
\downarrow \\
\Lambda
\end{array}\right)=\operatorname{Mimo} \tau_{\Lambda}\left(\begin{array}{c}
\Lambda \\
\downarrow \\
\Lambda / \mathbf{m}
\end{array}\right)=\operatorname{Mimo}\left(\begin{array}{c}
0 \\
\downarrow \\
\Lambda / \mathbf{m}
\end{array}\right) \cong\left(\begin{array}{c}
0 \\
\downarrow \\
k
\end{array}\right), \\
& \tau_{\mathcal{S}}\left(\begin{array}{c}
0 \\
\downarrow \\
k
\end{array}\right)=\operatorname{Mimo} \tau_{\Lambda}\left(\begin{array}{c}
k \\
\downarrow \\
k
\end{array}\right)=\left(\begin{array}{c}
k \\
\downarrow \\
k
\end{array}\right), \\
& \tau_{\mathcal{S}}\left(\begin{array}{c}
k \\
\downarrow \\
k
\end{array}\right)=\operatorname{Mimo} \tau_{\Lambda}\left(\begin{array}{c}
k \\
\downarrow \\
0
\end{array}\right)=\left(\begin{array}{c}
k \\
\downarrow \\
\Lambda
\end{array}\right) .
\end{aligned}
$$

So after six steps we are back to where we started. This is not a coincidence, as we will see in the next chapter.

Definition. Let $\mathcal{S}(\Lambda)_{\mathcal{I}}$ be the full subcategory of $\mathcal{S}(\Lambda)$ consisting of all objects which have no nonzero injective direct summands.

Corollary 5.3. Every object in $\mathcal{S}(\Lambda)_{\mathcal{I}}$ has the form $\operatorname{Mimo}(f)$ for some morphism $f: A \rightarrow B$ where the $\Lambda$-modules $A$ and $B$ have no nonzero injective direct summands.

Definition. By $\mathcal{H}^{\prime}(\Lambda)$ we denote the morphism category for $\underline{\bmod } \Lambda$. Thus, the objects are the morphisms $(\bar{f}: A \rightarrow B)$ in $\underline{\bmod } \Lambda$, and a morphism in $\mathcal{H}^{\prime}(\Lambda)$ from $(\bar{f}: A \rightarrow B)$ to $\left(\bar{f}^{\prime}: A^{\prime} \rightarrow B^{\prime}\right)$ is given by a pair $(\bar{a}, \bar{b})$ where $\bar{a}: A \rightarrow A^{\prime}$, $\bar{b}: B^{\prime} \rightarrow B$ are morphisms such that $\overline{f b}=\bar{a} \bar{f}^{\prime}$ holds in $\underline{\bmod } \Lambda$.

Corollary 5.4. The functor

$$
F: \mathcal{S}(\Lambda)_{\mathcal{I}} \longrightarrow \mathcal{H}^{\prime}(\Lambda), \quad f \mapsto \bar{f},
$$

is dense, preserves indecomposables, and reflects isomorphisms. Thus, F is a representation equivalence.

Proof. Clearly, $F$ is dense: If the object $\bar{f}$ in $\mathcal{H}^{\prime}(\Lambda)$ is represented by $f$, then $F \operatorname{Mimo}(f)$ and $\bar{f}$ are isomorphic in $\mathcal{H}^{\prime}(\Lambda)$.

The functor $F$ preserves indecomposables: Let $a$ be an indecomposable noninjective object in $\mathcal{S}(\Lambda)$. Then a occurs as the first term of an Auslander-Reiten sequence and hence has the form $a=\tau_{\mathcal{S}}(c)$ for some indecomposable nonprojective object $c \in \mathcal{S}(\Lambda)$. In particular, $F(a)=F \operatorname{Mimo}\left(\tau_{\Lambda} \operatorname{Cok}(c)\right)$ is isomorphic to $\tau_{\Lambda} \operatorname{Cok}(c)$ in $\mathcal{H}^{\prime}(\Lambda)$ which is an indecomposable object since Mimo is additive.

To show that $F$ reflects isomorphisms, let two objects $f, g \in \mathcal{S}(\Lambda)$ be given such that $F(f)$ and $F(g)$ are isomorphic in $\mathcal{H}^{\prime}(\Lambda)$. By Corollary 5.3, $f=\operatorname{Mimo}\left(f_{1}\right)$ 
and $g=\operatorname{Mimo}\left(g_{1}\right)$, where $f_{1}: A_{1} \rightarrow B_{1}$ and $g_{1}: C_{1} \rightarrow D_{1}$ are maps between $\Lambda$ modules with no nonzero injective direct summands. Since $\overline{f_{1}}$ and $\overline{g_{1}}$ are isomorphic in $\mathcal{H}^{\prime}(\Lambda)$ there are isomorphisms of $\Lambda$-modules $u: A_{1} \rightarrow C_{1}$ and $v: B_{1} \rightarrow D_{1}$ such that $f_{1} v-u g_{1}$ factorizes through an injective $\Lambda$-module. By Theorem 4.2, $f=\operatorname{Mimo}\left(f_{1}\right)$ and $g=\operatorname{Mimo}\left(g_{1}\right)$ are isomorphic.

One can go a little bit further.

Definition. Let $\mathcal{I}$ be the ideal in the category $\mathcal{S}(\Lambda)$ of all morphisms which factorize through an injective object in $\mathcal{S}(\Lambda)$. By $\mathcal{S}(\Lambda) / \mathcal{I}$ we denote the factor category of $\mathcal{S}(\Lambda)$ modulo $\mathcal{I}$. Clearly, the functor $F: \mathcal{S}(\Lambda) \rightarrow \mathcal{H}^{\prime}(\Lambda), f \mapsto \bar{f}$, annihilates every morphism in $\mathcal{I}$ and hence defines a functor $\bar{F}: \mathcal{S}(\Lambda) / \mathcal{I} \rightarrow \mathcal{H}^{\prime}(\Lambda)$.

Lemma 5.5. The functor $\bar{F}$ is full and dense.

Proof. Since the dense functor $F$ from Corollary 5.4 factorizes over $\bar{F}$, then $\bar{F}$ is also dense. To show that $\bar{F}$ is full, let $f: A \rightarrow B$ and $g: C \rightarrow D$ be objects in $\mathcal{S}(\Lambda)$, let $e: A \rightarrow I$ be an injective envelope and $e^{\prime}: B \rightarrow I$ an extension of $e$. If $(\bar{u}, \bar{v})$ is a morphism in $\operatorname{Hom}_{\mathcal{H}^{\prime}}(\bar{f}, \bar{g})$, then there are maps $u: A \rightarrow C, v: B \rightarrow D$, $w: I \rightarrow D$ such that $u g-f v=e w$. The following diagram is commutative:

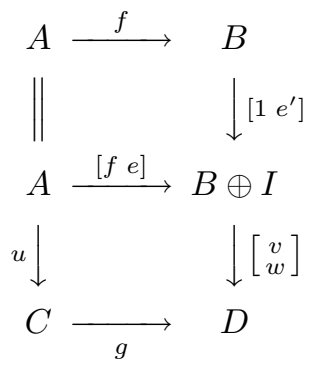

and hence represents two morphisms $f \stackrel{\left(1_{A},\left[1_{B} e^{\prime}\right]\right)}{\longrightarrow}[f e] \stackrel{\left(u,\left[\begin{array}{c}u \\ w\end{array}\right]\right)}{\longrightarrow} g$ in the category $\mathcal{S}(\Lambda)$. (Note that the first is a split monomorphism, so that $f$ and $[f e]$ become isomorphic objects in the factor category $\mathcal{S}(\Lambda) / \mathcal{I}$.) By applying the functor $F$ to the composition of the two morphisms, we obtain $(\bar{u}, \bar{v})$.

Remark. The following example shows that the functor $\bar{F}$ is not a categorical equivalence in general. Let $\Lambda$ be a uniserial ring of Loewy length 3 and $m$ a generator of the maximal ideal $\mathbf{m}$. Let $f$ be the object in $\mathcal{S}(\Lambda)$ given by the inclusion $f: \mathbf{m} / \mathbf{m}^{2} \rightarrow \Lambda / \mathbf{m}^{2}$. Then the multiplication $\mu_{m}$ by $m$ is a nonzero nilpotent endomorphism of $f$ which does not factorize through an injective object in $\mathcal{S}(\Lambda)$, but for which $F\left(\mu_{m}\right)=0$ holds. Thus, the functor $\bar{F}$ is not faithful.

\section{Morphisms in the Stable CATEGory}

In this section we assume that $\Lambda$ is a self-injective algebra. Then the stable category $\underline{\bmod } \Lambda$ modulo all morphisms which factorize through a projective-injective object, together with the suspension functor $\Omega^{-1}$, becomes a triangulated category. We recall the construction of triangles in this category, observe that the assignment $f \mapsto \operatorname{Cok} \operatorname{Mimo}(f)$ for a morphism $f: A \rightarrow B$ is related to the rotation of a triangle in the stable category, and retrieve the formula $\tau_{\mathcal{S}}^{6}(f) \cong f$ for the case that $\Lambda$ is a uniserial algebra. 
We recall from $[\mathrm{H}$, Chapter $\mathrm{I}]$ that the stable category $\underline{\bmod } \Lambda$ has the following standard triangles. For a morphism $f: A \rightarrow B$ in $\bmod \Lambda$, take the short exact sequence given by the inclusion of $A$ in its injective envelope, $i: A \rightarrow \mathrm{I}(A)$, and the cokernel map $c: \mathrm{I}(A) \rightarrow \Omega^{-1} A$, and form the push-out diagram along $f$ :

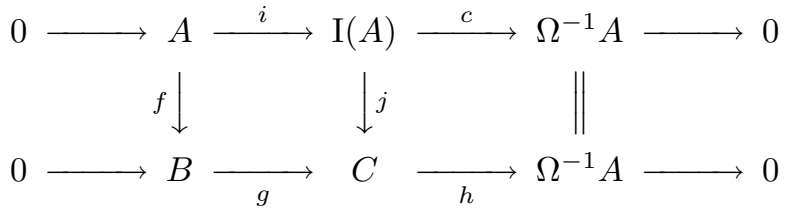

Then the standard triangle corresponding to $f$ is

$$
T(f): \quad A \stackrel{\bar{f}}{\longrightarrow} B \stackrel{\bar{g}}{\longrightarrow} C \stackrel{\bar{h}}{\longrightarrow} \Omega^{-1} A
$$

and each triangle in the stable category is isomorphic to a standard one $[\mathrm{H}$, Theorem I.2.6].

A morphism between triangles $T: A \rightarrow B \rightarrow C \rightarrow \Omega^{-1} A$ and $T^{\prime}: A^{\prime} \rightarrow B^{\prime} \rightarrow$ $C^{\prime} \rightarrow \Omega^{-1} A^{\prime}$ consists of morphisms $\bar{a}, \bar{b}, \bar{c}$ in the stable category which make the following diagram commutative:

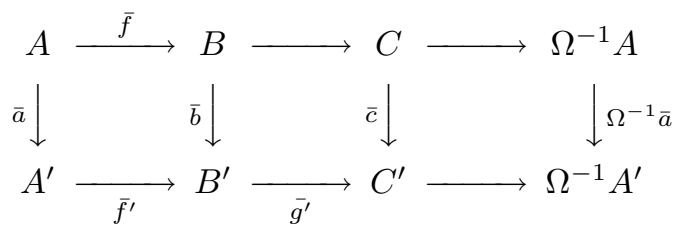

The two triangles are isomorphic if $\bar{a}, \bar{b}$, and $\bar{c}$ are isomorphisms. From [H, I.1.2 and I.1.6] we recall that for the existence of a morphism between the triangles it is sufficient to have a map $b: B \rightarrow B^{\prime}$ such that $\overline{f b g^{\prime}}=0$. Moreover, a morphism $(\bar{a}, \bar{b}, \bar{c})$ is an isomorphism if $\bar{a}$ and $\bar{b}$ are isomorphisms in $\underline{\bmod } \Lambda$. Thus, isomorphisms between triangles are obtained from pairs $(\bar{a}, \bar{b})$ of isomorphisms which make the left square in the above diagram commutative. This is to say that if $(\bar{a}, \bar{b}):(A \stackrel{\bar{f}}{\rightarrow} B) \rightarrow\left(A^{\prime} \stackrel{\bar{f}^{\prime}}{\rightarrow} B^{\prime}\right)$ is an isomorphism in the category $\mathcal{H}^{\prime}(\Lambda)$ of morphisms in the stable category $\underline{\bmod } \Lambda$, then the triangles $T(f)$ and $T\left(f^{\prime}\right)$ are isomorphic.

Given a triangle $T: A \stackrel{\bar{f}}{\rightarrow} B \stackrel{\bar{g}}{\rightarrow} C \stackrel{\bar{h}}{\rightarrow} \Omega^{-1} A$, then the rotation of $T$ below is also a triangle, as required by the axioms of a triangulated category.

$$
T^{\mathrm{R}}: \quad B \stackrel{\bar{g}}{\rightarrow} C \stackrel{\bar{h}}{\rightarrow} \Omega^{-1} A \stackrel{-\Omega^{-1}}{\longrightarrow} \Omega^{-1} B .
$$

This operation $T(f) \mapsto T(f)^{\mathrm{R}}$ in the triangulated category gives rise to a selfequivalence $\bar{f} \mapsto \bar{f}^{\mathrm{R}}$ on the category $\mathcal{H}^{\prime}(\Lambda)$ of morphisms in the stable category. According to the following lemma, a map $g$ in $\bmod \Lambda$ representing $\bar{f}^{\mathrm{R}}$ in $\underline{\bmod } \Lambda$ is obtained as $g=$ Cok Mimo $f$.

Lemma 6.1. For a map $f: A \rightarrow B$ in $\bmod \Lambda$, the two morphisms $\bar{f}^{\mathrm{R}}$ and $\overline{\text { Cok Mimo } f}$ are isomorphic in $\mathcal{H}^{\prime}(\Lambda)$. 
Proof. In the following diagram with exact rows, the top row defines the cokernel map for $\operatorname{Mimo}(f)=\left[\begin{array}{ll}f & e\end{array}\right]$, while the third row is given by the push-out diagram defining $T(f)$ :

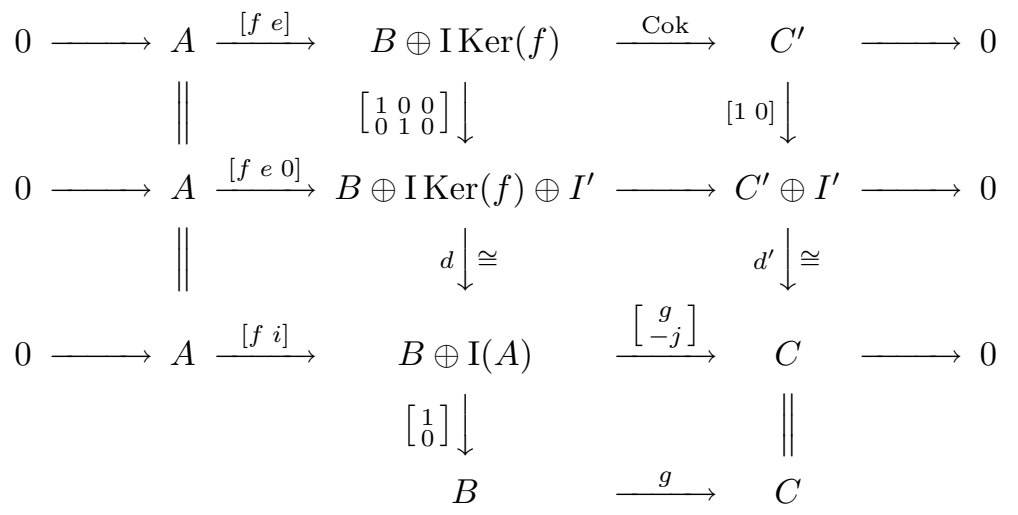

All squares with the possible exception of the square at the bottom are commutative: Since the map $[f i]$ is a monomorphism, it follows from Claim 2 in Chapter 4 that there is an injective module $I^{\prime}$ and an isomorphism $d$ such that the left square between the second and third row is commutative; if $d^{\prime}$ is the cokernel map, then both squares are commutative. Here the exact sequence in the first row is a direct summand of the sequence in the second row, the complement being the sequence $0 \rightarrow 0 \rightarrow I^{\prime} \stackrel{1}{\rightarrow} I^{\prime} \rightarrow 0$. Finally, the map $g$ which represents the second map in the triangle $T(f)$ occurs as a restriction of the cokernel map in the third row.

Note that all vertical maps become isomorphisms when considered in the stable category. Thus, the two morphisms $\overline{\mathrm{Cok}}: B \oplus \operatorname{I} \operatorname{Ker}(f) \rightarrow C^{\prime}$ and $\bar{g}: B \rightarrow C$ are isomorphic when considered as objects in $\mathcal{H}^{\prime}(\Lambda)$.

Since $\Lambda$ is a self-injective algebra, there is a third self-equivalence on the morphism category $\mathcal{H}^{\prime}(\Lambda)$ (besides the suspension and the rotation) given by the Auslander-Reiten translation $\tau_{\Lambda}$. According to [ARS, Proposition IV.3.7], the functors $\tau_{\Lambda}$ and $\mathcal{N} \Omega^{2}$ from $\underline{\bmod } \Lambda$ to $\underline{\bmod } \Lambda$ are isomorphic, where $\mathcal{N}=D \operatorname{Hom}_{\Lambda}(-, \Lambda)$ is the Nakayama automorphism. It follows that the Auslander-Reiten translation $\tau_{\Lambda}$ preserves triangles. As a consequence, the functor $\tau_{\Lambda}$ commutes with the rotation $\bar{f} \mapsto \bar{f}^{\mathrm{R}}$, and also with the suspension $\Omega^{-1}$ in the sense that for each morphism $f: A \rightarrow B$ there is a commutative diagram in which the vertical maps are isomorphisms:

$$
\begin{aligned}
\tau_{\Lambda} \Omega^{-1} A \stackrel{\tau_{\Lambda} \Omega^{-1} f}{\longrightarrow} \tau_{\Lambda} \Omega^{-1} B \\
\eta^{\prime}(f) \downarrow \cong \quad \eta^{\prime \prime}(f) \mid \cong \\
\Omega^{-1} \tau_{\Lambda} A \underset{\Omega^{-1} \tau_{\Lambda} f}{\longrightarrow} \Omega^{-1} \tau_{\Lambda} B
\end{aligned}
$$

The self-equivalence on the triangulated category given by the rotation $T \mapsto T^{\mathrm{R}}$ yields isomorphisms $T^{3 \mathrm{R}} \cong-\Omega^{-1} T$ and $T^{6 \mathrm{R}} \cong \Omega^{-2} T$. We obtain the following consequence for the Auslander-Reiten translation $\tau_{\mathcal{S}}$ in the submodule category. 
Theorem 6.2. Suppose $\Lambda$ is a self-injective algebra. If $(f: A \rightarrow B)$ is an indecomposable nonprojective object in $\mathcal{S}(\Lambda)$, then there are the following isomorphisms in the morphism category $\mathcal{H}^{\prime}(\Lambda)$ :

$$
\begin{aligned}
& \overline{\tau_{\mathcal{S}}(f)} \cong \tau_{\Lambda}(\overline{\operatorname{Cok} f}), \\
& \overline{\tau_{\mathcal{S}}^{3}(f)} \cong-\tau_{\Lambda}^{3} \Omega^{-1}(\bar{f}), \text { and } \\
& \overline{\tau_{\mathcal{S}}^{6}(f)} \cong \tau_{\Lambda}^{6} \Omega^{-2}(\bar{f}) .
\end{aligned}
$$

Corollary 6.3. Under the assumptions of the theorem, there are the following isomorphisms in the submodule category $\mathcal{S}(\Lambda)$ :

$$
\tau_{S}^{3}(f) \cong-\operatorname{Mimo} \tau_{\Lambda}^{3} \Omega^{-1}(f), \quad \tau_{S}^{6}(f) \cong \operatorname{Mimo} \tau_{\Lambda}^{6} \Omega^{-2}(f) .
$$

Proof of Corollary 6.3. The functor $\mathcal{S}(\Lambda)_{I} \rightarrow \mathcal{H}^{\prime}(\Lambda), f \mapsto \bar{f}$ in Corollary 5.3 reflects isomorphisms, so the assertion follows from Theorem 6.2.

Proof of Theorem 6.2. The first statement (1) follows from Theorem 5.1 since any map $g$ is stably equivalent to $\operatorname{Mimo}(g)$. For the proof of assertion (2) we use Theorem 5.1 to compute

$$
\tau_{\mathcal{S}}^{3}(A \stackrel{f}{\rightarrow} B)=\operatorname{Mimo} \tau_{\Lambda} \operatorname{Cok} \operatorname{Mimo} \tau_{\Lambda} \operatorname{Cok} \operatorname{Mimo} \tau_{\Lambda} \operatorname{Cok}(f) .
$$

Then we obtain the following isomorphisms of objects in $\mathcal{H}^{\prime}(\Lambda)$ :

$$
\begin{aligned}
\overline{\tau_{\mathcal{S}}^{3}(f)} \cong \tau_{\Lambda}\left(\overline{\operatorname{Cok} \operatorname{Mimo} \tau_{\Lambda} \operatorname{Cok} \operatorname{Mimo} \tau_{\Lambda} \operatorname{Cok} f}\right) \\
\cong \tau_{\Lambda}\left({\overline{\tau_{\Lambda} \operatorname{Cok} \operatorname{Mimo} \tau_{\Lambda} \operatorname{Cok} f}}^{\mathrm{R}}\right) \\
\cong \tau_{\Lambda}^{2}\left({\overline{\operatorname{CokMimo} \tau_{\Lambda} \operatorname{Cok} f}}^{\mathrm{R}}\right) \\
\cong \tau_{\Lambda}^{2}\left({\overline{\tau_{\Lambda} \operatorname{Cok} f}}^{2 \mathrm{R}}\right) \\
\cong \tau_{\Lambda}^{3}\left(\overline{\operatorname{Cokf}}^{2 \mathrm{R}}\right) \\
\cong \tau_{\Lambda}^{3}\left(\bar{f}^{3 \mathrm{R}}\right) \\
\cong \tau_{\Lambda}^{3}\left(-\Omega^{-1}(\bar{f})\right)
\end{aligned}
$$

where the first isomorphism is justified by (1), the second, fourth, and sixth isomorphisms follow from Lemma 6.1, the third and fifth equalities come from the commutativity of $\tau_{\Lambda}$ with the rotation, and the last map is an isomorphism since a threefold rotation of a triangle is obtained by applying the functor $-\Omega^{-1}$.

In order to deduce the third assertion from the second, pick a representative map $g: \Omega^{-1} A \rightarrow \Omega^{-1} B$ in $\bmod \Lambda$ for the morphism $\overline{\tau_{\mathcal{S}}^{3}(f)}$ in $\underline{\bmod } \Lambda$ such that $\Omega^{-1} A$ and $\Omega^{-1} B$ have no nonzero injective direct summands. Then $\operatorname{Mimo}(g)$ is an indecomposable nonprojective object in $\mathcal{S}(\Lambda)$, and by Proposition 4.1 its isomorphism class does not depend on the choice of the map $g$. The following morphisms in $\underline{\bmod } \Lambda$ are isomorphic objects in $\mathcal{H}^{\prime}(\Lambda)$ :

$$
\overline{\tau_{\mathcal{S}}^{6}(f)} \cong \overline{\tau_{\mathcal{S}}^{3} \operatorname{Mimo}(g)} \cong-\tau_{\Lambda}^{3} \Omega^{-1}(\bar{g}) \cong-\tau_{\Lambda}^{3} \Omega^{-1}\left(-\tau_{\Lambda}^{3} \Omega^{-1}(\bar{f})\right) \cong \tau_{\Lambda}^{6} \Omega^{-2}(\bar{f}) .
$$

We conclude this section with three applications. 
Corollary 6.4. Suppose $\Lambda$ is a self-injective algebra such that $\tau_{\Lambda}$ coincides with $\Omega^{2}$. If $(f: A \rightarrow B)$ is an indecomposable nonprojective object in $\mathcal{S}(\Lambda)$, then there is an isomorphism of objects

$$
\tau_{\mathcal{S}}^{3}(f) \cong-\operatorname{Mimo} \Omega^{5}(f)
$$

in $\mathcal{S}(\Lambda)$.

Proof. Since $\tau_{\Lambda}$ coincides with $\Omega^{2}$, we can simplify the expression in formula (2) of Theorem 6.2 and see that

$$
\overline{\tau_{\mathcal{S}}^{3}(f)} \cong-\tau_{\Lambda}^{3} \Omega^{-1}(\bar{f}) \cong-\Omega^{6} \Omega^{-1}(\bar{f}) \cong-\Omega^{5}(\bar{f})
$$

in $\mathcal{H}^{\prime}(\Lambda)$. The functor $\mathcal{S}(\Lambda)_{\mathcal{I}} \rightarrow \mathcal{H}^{\prime}(\Lambda), f \mapsto \bar{f}$, in Corollary 5.3 reflects isomorphisms, so $\tau_{\mathcal{S}}^{3}(f)$ and $-\Omega^{5}(f)$ are isomorphic in $\mathcal{S}(\Lambda)$.

Note that for any symmetric algebra, the functors $\tau_{\Lambda}$ and $\Omega^{2}$ coincide (see for example [ARS, Proposition IV.3.8]); thus we can apply Corollary 6.4 in this case.

Corollary 6.5. Let $\Lambda$ be a commutative uniserial algebra. Then for an indecomposable nonprojective object $(f: A \rightarrow B)$ in $\mathcal{S}(\Lambda)$, there is an isomorphism of objects

$$
\tau_{\mathcal{S}}^{6}(f) \cong f
$$

in $\mathcal{S}(\Lambda)$.

Proof. Since $\Lambda$ is a commutative uniserial algebra, all the functors $\tau_{\Lambda}, \Omega^{2}$ and $\Omega^{-2}$ are equivalent to the identity functor on $\underline{\bmod } \Lambda$, thus Corollary 6.4 shows that $\tau_{\mathcal{S}}^{6}(f)$ and $\Omega^{10}(f) \cong f$ are isomorphic objects of $\mathcal{S}(\Lambda)$.

Definition. By $\mathbb{A}_{\infty}^{\infty}$ we denote the doubly infinite linear quiver

$$
\cdots \quad \stackrel{\alpha}{\longleftarrow} \bullet^{-1} \stackrel{\alpha}{\longleftarrow} \bullet^{0} \stackrel{\alpha}{\longleftarrow} \bullet^{1} \stackrel{\alpha}{\longleftarrow} \quad \ldots .
$$

The path algebra $k \mathbb{A}_{\infty}^{\infty}$ of this quiver is the associative $k$-algebra with basis the paths in $\mathbb{A}_{\infty}^{\infty}$. If $\alpha^{n}$ denotes the ideal spanned by all paths of length at least $n$, then the factor algebra $\Lambda=k \mathbb{A}_{\infty}^{\infty} / \alpha^{n}$ is a locally bounded associative $k$-algebra. A $\Lambda$-module $A$ consists of a sequence $\left(A_{i}\right)_{i \in \mathbb{Z}}$ of $k$-modules together with a sequence $\left(\alpha_{i}: A_{i} \rightarrow A_{i-1}\right)_{i \in \mathbb{Z}}$ of linear maps. By $A[\ell]$ we denote the shifted module given by the spaces $\left(A_{i-\ell}\right)_{i}$ and the maps $\left(\alpha_{i-\ell}\right)_{i}$.

Corollary 6.6. Let $\Lambda$ be the associative algebra $k \mathbb{A}_{\infty}^{\infty} / \alpha^{n}$ where $k$ is a field. For an indecomposable nonprojective object $(f: A \rightarrow B)$ in $\mathcal{S}(\Lambda)$, the following formula holds:

$$
\tau_{\mathcal{S}}^{6}(f) \cong f[n-6]
$$

Proof. The Auslander-Reiten translation $\tau_{\Lambda}$ is given by the shift $A \mapsto A[-1]$ along the arrow $\alpha$, hence the functor $\tau_{\Lambda}$ on the stable category $\underline{\bmod } \Lambda$ preserves triangles. Also, $\tau_{\Lambda}$ commutes with $\Omega^{-1}$ and with the rotation in a triangle. Moreover, for a nonprojective indecomposable $\Lambda$-module $A$, the process of taking the cokernel of 
the injective envelope twice yields the module $A[n]$. With these adjustments, the claim follows from (3) in Theorem 6.2 as in the proof of Corollary 6.4.

\section{Auslander-Reiten sequences}

In this section we show that "most" Auslander-Reiten sequences in the category $\mathcal{S}(\Lambda)$ become split exact sequences in the category $\bmod \Lambda$, when restricted to the short exact sequence of the submodules, or to the short exact sequence of the big modules. We describe the exceptions in detail. The remaining sink and source maps are associated with the projective and the injective objects and have been specified in Chapter 1 . We only need to assume here that $\Lambda$ is a locally bounded associative algebra. First we deal with the exceptions.

Proposition 7.1 (Auslander-Reiten sequences with components not split exact). Let $0 \rightarrow A \stackrel{f}{\rightarrow} B \stackrel{g}{\rightarrow} C \rightarrow 0$ be an Auslander-Reiten sequence in $\bmod \Lambda$.

1. The Auslander-Reiten sequence in $\mathcal{S}(\Lambda)$ ending at $(0 \rightarrow C)$ has the form

$$
0 \longrightarrow{ }_{A}^{A} 1 \stackrel{1}{\longrightarrow} \quad \underset{B}{\stackrel{A}{\perp}} \stackrel{0}{g} \underset{C}{\stackrel{\downarrow}{C}} \longrightarrow 0 .
$$

2. With $e: A \rightarrow \mathrm{I}(A)$ an injective envelope, the Auslander-Reiten sequence in $\mathcal{S}(\Lambda)$ ending at $\left(1_{C}: C \rightarrow C\right)$ has the form

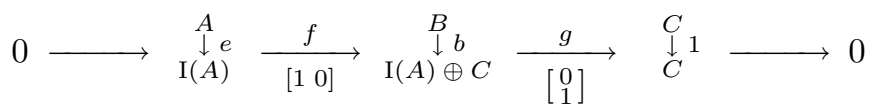

where $b$ is the map $\left[e^{\prime} g\right]$ with $e^{\prime}: B \rightarrow \mathrm{I}(A)$ an extension of the map $e$.

Proof. 1. We show that the map $(0, g)$ is minimal right almost split. Clearly, this map is right minimal and not a split epimorphism. In order to show that $(0, g):(A \stackrel{f}{\rightarrow} B) \rightarrow(0 \rightarrow C)$ is right almost split, let $\left(t^{\prime}, t\right):\left(x^{\prime}: X^{\prime} \rightarrow X\right) \rightarrow$ $(0 \rightarrow C)$ be a test map which is not a split epimorphism. Then $t: X \rightarrow C$ is not a split epimorphism in $\bmod \Lambda$, so there is $u: X \rightarrow B$ such that $t=u g$. Since the composition $X^{\prime} \stackrel{x^{\prime}}{\rightarrow} X \stackrel{u}{\rightarrow} B \stackrel{g}{\rightarrow} C$ is zero, there is $u^{\prime}: X^{\prime} \rightarrow A$ such that $x^{\prime} u=u^{\prime} f$. Thus, $\left(u^{\prime}, u\right)$ is a morphism which satisfies $\left(t^{\prime}, t\right)=\left(u^{\prime}, u\right)(0, g)$.

2. A straightforward argument shows that the map $\left(g,\left[\begin{array}{l}0 \\ 1\end{array}\right]\right)$ is right minimal. We verify that a test map $\left(t^{\prime}, t\right):\left(x^{\prime}: X^{\prime} \rightarrow X\right) \rightarrow\left(1_{C}: C \rightarrow C\right)$ which is not a split epimorphism factorizes over $\left(g,\left[\begin{array}{l}0 \\ 1\end{array}\right]\right)$. Since $t^{\prime}: X^{\prime} \rightarrow C$ is not a split epimorphism, there is $u^{\prime}: X^{\prime} \rightarrow B$ such that $t^{\prime}=u^{\prime} g$. Let $u_{1}: X \rightarrow \mathrm{I}(A)$ be an extension of $u^{\prime} e^{\prime}: X^{\prime} \rightarrow \mathrm{I}(A)$ to $X$ and put $u=\left[u_{1} t\right]: X \rightarrow \mathrm{I}(A) \oplus C$. Then $\left(u^{\prime}, u\right)$ is a morphism in $\mathcal{S}(\Lambda)$ such that $\left(t^{\prime}, t\right)=\left(u^{\prime}, u\right)\left(g,\left[\begin{array}{l}0 \\ 1\end{array}\right]\right)$

The remaining Auslander-Reiten sequences in $\mathcal{S}(\Lambda)$ are made up from two split exact sequences.

Proposition 7.2 (Auslander-Reiten sequences with components split exact). Suppose that $\left(c: C^{\prime} \rightarrow C\right)$ is an indecomposable object in $\mathcal{S}(\Lambda)$ such that the morphism $c$ in $\bmod \Lambda$ is not split monic. If

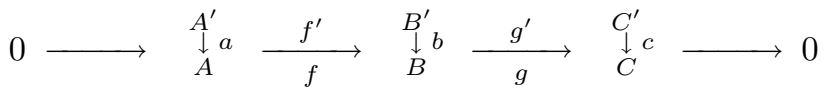


is an Auslander-Reiten sequence in $\mathcal{S}(\Lambda)$, then both sequences in $\bmod \Lambda$,

$$
0 \rightarrow A^{\prime} \stackrel{f^{\prime}}{\rightarrow} B^{\prime} \stackrel{g^{\prime}}{\rightarrow} C^{\prime} \rightarrow 0 \quad \text { and } \quad 0 \rightarrow A \stackrel{f}{\rightarrow} B \stackrel{g}{\rightarrow} C \rightarrow 0,
$$

are split exact.

Proof. Since the map $c$ is not a split monomorphism, the test maps

$$
(0,1):(0 \rightarrow C) \rightarrow\left(C^{\prime} \stackrel{c}{\rightarrow} C\right) \text { and }(1, c):\left(C^{\prime} \stackrel{1}{\rightarrow} C^{\prime}\right) \rightarrow\left(C^{\prime} \stackrel{c}{\rightarrow} C\right)
$$

are not split epimorphisms and hence factorize over $\left(g^{\prime}, g\right)$. Thus, both $g$ and $g^{\prime}$ are split epimorphisms.

We combine this result with Theorem 5.1.

Corollary 7.3 (The middle term of an Auslander-Reiten sequence). Let ( $c: C^{\prime} \rightarrow$ $C)$ be an indecomposable object in $\mathcal{S}(\Lambda)$ such that the morphism $c$ in $\bmod \Lambda$ is not split monic.

1. The Auslander-Reiten sequence ending in $\left(c: C^{\prime} \rightarrow C\right)$ has the form

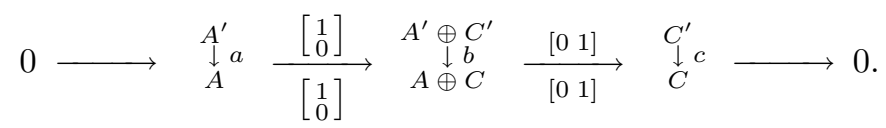

2. The map $b$ defining the middle term is given as follows by a map $h: C^{\prime} \rightarrow A$ :

$$
b=\left(\begin{array}{ll}
a & 0 \\
h & c
\end{array}\right): A^{\prime} \oplus C^{\prime} \rightarrow A \oplus C .
$$

3. The first term $\left(A^{\prime} \stackrel{a}{\rightarrow} A\right)=\tau_{\mathcal{S}}\left(C^{\prime} \stackrel{c}{\rightarrow} C\right)$ of the Auslander-Reiten sequence ending in $\left(c: C^{\prime} \rightarrow C\right)$ is isomorphic to $\operatorname{Mimo} \tau_{\Lambda} \operatorname{Cok}(c)$, in particular, $A^{\prime}=\tau_{\Lambda} C$ and $A=\tau_{\Lambda} C^{\prime \prime} \oplus I$ where $I$ is an injective $\Lambda$-module and $C^{\prime \prime}=\operatorname{Cok}(c)$.

To conclude this chapter, we state the following dual results for the category $\mathcal{F}(\Lambda)$.

Proposition 7.4 (AR-sequences in $\mathcal{F}(\Lambda)$ with components not split exact). Suppose that $0 \rightarrow A \rightarrow B \rightarrow C \rightarrow 0$ is an Auslander-Reiten sequence in $\bmod \Lambda$.

1. The Auslander-Reiten sequence in $\mathcal{F}(\Lambda)$ starting at $(A \rightarrow 0)$ has the form

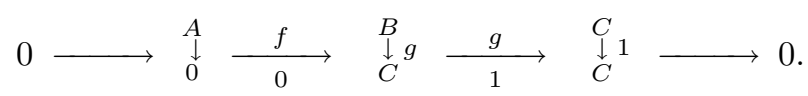

2. The Auslander-Reiten sequence in $\mathcal{F}(\Lambda)$ starting at $\left(1_{A}: A \rightarrow A\right)$ has the form

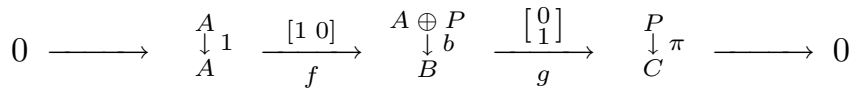

where $\pi: P \rightarrow C$ is a projective cover, and where the map $b$ has the form $b=[f \hat{\pi}]^{t}$ with $\hat{\pi}: P \rightarrow B$ a lifting of $\pi$ to $B$.

Proposition 7.5 (AR-sequences in $\mathcal{F}(\Lambda)$ with split exact components). Suppose that $\left(a: A \rightarrow A^{\prime \prime}\right)$ is indecomposable in $\mathcal{F}(\Lambda)$ such that the epimorphism a is not a split epimorphism. If

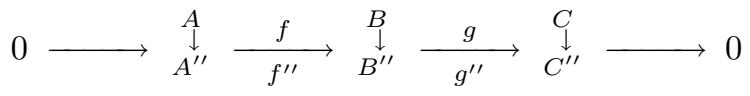


is an Auslander-Reiten sequence in $\mathcal{F}(\Lambda)$, then both sequences in $\bmod \Lambda$,

$$
0 \rightarrow A \stackrel{f}{\rightarrow} B \stackrel{g}{\rightarrow} C \rightarrow 0 \quad \text { and } \quad 0 \rightarrow A^{\prime \prime} \stackrel{f^{\prime \prime}}{\rightarrow} B^{\prime \prime} \stackrel{g^{\prime \prime}}{\rightarrow} C^{\prime \prime} \rightarrow 0,
$$

are split exact.

Corollary 7.6 (The middle term of an AR-sequence in $\mathcal{F}(\Lambda))$. Let $\left(a: A \rightarrow A^{\prime \prime}\right)$ be an indecomposable object in $\mathcal{F}(\Lambda)$ such that the map a in $\bmod \Lambda$ is not a split epimorphism.

1. The Auslander-Reiten sequence starting at $\left(a: A \rightarrow A^{\prime \prime}\right)$ has the form

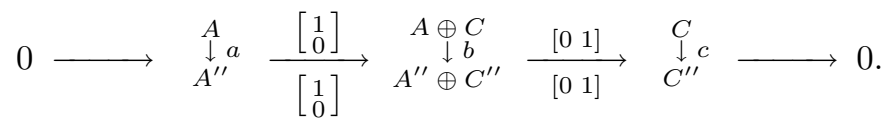

2. The morphism $b$ defining the middle term is given as follows by some map $h: C \rightarrow A^{\prime \prime}:$

$$
b=\left(\begin{array}{ll}
a & 0 \\
h & c
\end{array}\right): A \oplus C \rightarrow A^{\prime \prime} \oplus C^{\prime \prime} .
$$

3. The last term $\left(C \stackrel{c}{\rightarrow} C^{\prime \prime}\right)=\tau_{\mathcal{F}}^{-}\left(A \stackrel{a}{\rightarrow} A^{\prime \prime}\right)$ of the Auslander-Reiten sequence starting in $\left(a: A \rightarrow A^{\prime \prime}\right)$ is isomorphic to Mepi $\tau_{\Lambda}^{-} \operatorname{Ker}(a)$, in particular, $C=$ $\tau_{\Lambda}^{-} A^{\prime} \oplus P$ and $C^{\prime \prime}=\tau_{\Lambda}^{-} A$ where $P$ is a projective $\Lambda$-module and $A^{\prime}=\operatorname{Ker}(a)$.

\section{REFERENCES}

[ARS] M. Auslander, I. Reiten, S. O. Smalø: Representation Theory of Artin Algebras. Cambridge University Press (1995). MR1314422 (96c:16015)

[AS] M. Auslander, S. O. Smalø: Almost split sequences in subcategories, Journal of Algebra, 69 (1981), 426-454. MR617088 (82j:16048a)

[GR] P. Gabriel and A. V. Roĭter: Representations of finite dimensional algebras (with a chapter by Bernhard Keller), in: Encyclopedia of mathematical Sciences 73, Algebra VIII, 1-177, Springer, Berlin 1992. MR1239446 (94h:16001a)

[H] D. Happel: Triangulated Categories in the Representation Theory of Finite Dimensional Algebras, London Mathematical Society Lecture Notes series 119, ix+208pp, Cambridge University Press, Cambridge 1988. MR935124 (89e:16035)

[RS1] C.M. Ringel, M. Schmidmeier: Invariant subspaces of nilpotent linear operators. I, J. Reine Angew. Mathematik (Crelle) (to appear), 1-55.

[RS2] C.M. Ringel, M. Schmidmeier: Submodule categories of wild representation type, Journal for Pure and Applied Algebra 205 (2006), 412-422. MR2203624 (2006i:16025)

[S] A. Skowroński: Tame triangular matrix algebras over Nakayama algebras, J. London Math. Soc. 34 (1986), 245-264. MR856509 (87m:16046)

Fakultät für Mathematik, Universität Bielefeld, P.O. Box 100 131, D-33 501 BieleFELD, GERMANY

E-mail address: ringel@math.uni-bielefeld.de

Department of Mathematical Sciences, Florida Atlantic University, Boca Raton, FLORIDA 33431-0991

E-mail address: markus@math.fau.edu 